\title{
NORMAL FORM FOR SEMI-LINEAR KLEIN-GORDON EQUATIONS WITH SUPERQUADRATIC OSCILLATORS
}

\author{
BY RAFIK IMEKRAZ
}

\begin{abstract}
. -
We prove a long time existence result for semi-linear Klein-Gordon equations with a superquadratic potential for small and smooth initial datum. The proof relies on a weak separation of eigenvalues and specific multilinear estimates of eigenfunctions of superquadratic oscillators.
\end{abstract}

RÉSUMÉ (Equations de Klein-Gordon semi-linéaires avec des oscillateurs surquadratiques)

Nous prouvons un résultat d'existence, sur des grands temps, pour des équations semi-linéaires de Klein-Gordon avec un potentiel surquadratique pour des petites conditions initiales régulières. La preuve repose sur une faible séparation des valeurs propres et des estimations spécifiques des modes propres des oscillateurs surquadratiques.

\section{Contents}

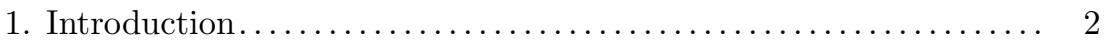

2. Spectral analysis................................. 9

3. Multilinear models............................... 14

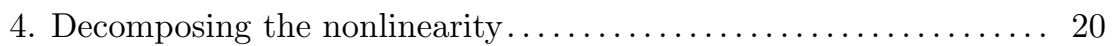

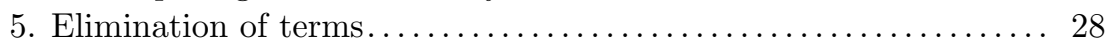

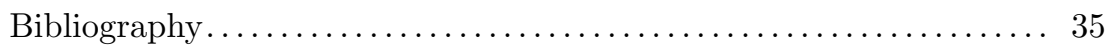

February 27, 2013

RAFik Imekraz, Université de Bordeaux 1, Institut de Mathématiques de Bordeaux UMR 5251, 351, cours de la Libération F 33405 TALENCE cedex FRANCE

E-mail : rafik.imekraz@math.u-bordeaux1.fr

Key words and phrases. - Normal forms, superquadratic, Klein-Gordon, semi-linear. 


\section{Introduction}

1.1. Context and statement of the result. - In this paper, we are interested with the Klein-Gordon equation on the real line with a superquadratic potential

$$
\left(\partial_{t}^{2}-\partial_{x}^{2}+x^{2 \alpha}+m^{2}\right) w=w^{n+1}, \quad(x, t) \in \mathbb{R}^{2} .
$$

Here, the nonlinearity $w^{n+1}$ is at least quadratic $(n \geq 1)$. The integer $\alpha$ will satisfy $\alpha \geq 2$ in our results but we will talk about the case $\alpha=1$ in this introduction. For any $s>0$, the natural Sobolev space of the equation (1) is given by

$$
\mathcal{H}_{\alpha}^{s}=\operatorname{Dom}\left(-\partial_{x}^{2}+x^{2 \alpha}\right)^{\frac{s}{2}}
$$

It turns out that the previous space is quite well understood as a functional space (see for instance [YZ04]) and is an algebra for $s>\frac{1}{2}$ :

$$
\mathcal{H}_{\alpha}^{s}=\left\{f \in \mathcal{D}^{\prime}(\mathbb{R}), \quad\|f\|_{H^{s}}+\left\|x^{\alpha s} f(x)\right\|_{L^{2}(\mathbb{R})}<+\infty\right\} .
$$

For the equation (1) and for any $s>\frac{1}{2}$, the local theory asserts that for a small initial datum $w(0, \cdot) \times \dot{w}(0, \cdot) \in \mathcal{H}_{\alpha}^{s+1} \times \mathcal{H}_{\alpha}^{s}$ such that $\|w(0, \cdot)\|_{\mathcal{H}_{\alpha}^{s+1}}+$ $\|\dot{w}(0, \cdot)\|_{\mathcal{H}_{\alpha}^{s}}=\varepsilon \ll 1$ holds, the corresponding solution is bounded by $K \varepsilon$, in the sense that $\|w(t, \cdot)\|_{\mathcal{H}_{\alpha}^{s+1}}+\|\dot{w}(t, \cdot)\|_{\mathcal{H}_{\alpha}^{s}} \leq K \varepsilon$, on a long time of order $\varepsilon^{-n}$. The time $\varepsilon^{-n}$ is called the local existence time for the nonlinearity $w^{n+1}$. It is a natural question to ask if one can improve the local existence time. Such a question has been studied by several authors for the nonlinear Klein-Gordon

$$
\left(\partial_{t}^{2}-\Delta_{g}+V(x)+m^{2}\right) w=N L(w), \quad x \in X .
$$

The first paper in this direction is probably [Bou96] which considers $X=S^{1}$ with a Hamiltonian nonlinearity. In the last decade Bambusi and Grébert proved that the Hamiltonian structure allows an iterative normal form procedure to improve the local existence time (see [Bam03, Gré07, Bam07, BG04, BG06]). Speaking roughly, making a normal form for an equation is similar to increase the degree of the nonlinearity. On the other part, Delort and Szeftel dealt with more general nonlinearities (with derivatives in $x$ and $t$ ) on the sphere and radial manifolds ([DS04, DS06a, DS06b]). Bambusi, Delort, Grébert and Szeftel unified their point of view in the paper [BDGS07] (see also [Ime13] for a generalization if $X$ is a Toeplitz structure with an adequate periodicity assumption). In previous papers, the operator $\sqrt{-\Delta_{g}+V}$ on the manifold $X$ (for instance $S^{d}$ ) has a separated spectrum in the sense that the difference of two different eigenvalues is uniformly bounded from below (more generally there is a "packet separation" on a Zoll manifold). In those situations, it turns out that if the nonlinearity makes the equation to be Hamiltonian, the local existence time can be improved in $C(r) \varepsilon^{-r}$ for any $r>1$, we usually say that the solutions admit almost global existence. 
Of course, there are also manifolds for which the spectrum of $\sqrt{-\Delta_{g}+V}$ is not separated. Simplest cases are probably tori, of dimension larger than 2 , endowed with their usual riemannian structures. When the spectrum is not separated, Delort introduced a normal form approach in [Del09] which gives a way to improve the local existence time for tori $\mathbb{T}^{d}$. See also an improvement if $d \geq 4$ in [FZ10] by Fang and Zhang.

When the spectrum is not separated, Delort's approach allows to hope that the local existence time is at least of order $\varepsilon^{-A n}$ where the number $A>1$ is explicit.

Let us go back to the equation (1). The case $\alpha=1$, which so deals with the quantum harmonic oscillator $-\partial_{x}^{2}+x^{2}$, has been considered by Zhang in the paper [Zha10]. The local existence time is proved to be at least $\varepsilon^{-\frac{25 n}{18}}$ (notice that $\frac{25}{18}<2$ ). It is highly tempting to think the case $\alpha=+\infty$ as the semi-linear Klein-Gordon equation with the operator $\sqrt{-\Delta}$ on $]-1,+1[$ with Dirichlet conditions. This case has for instance been considered in [BG06] and almost global existence is obtained. For this reason, it is natural to expect that it is possible to reach for (1) a local existence time of order $\varepsilon^{-n A(\alpha)}$ where $(A(\alpha))_{\alpha \geq 1}$ is an increasing sequence such that $\lim _{\alpha \rightarrow+\infty} A(\alpha)=+\infty$. Our work, which has been highly motivated by the paper [Zha10], gives a result in this direction. Precisely, we can improve the local existence time to $\varepsilon^{-2 n}$ for the equation (1) if $\alpha \geq 4$. In the paper [Zha10] (as well in [Del09, FZ10]), the obstruction $A<2$ in the local existence time $\varepsilon^{-n A}$ comes from the fact that a complete normal form seems not to be possible with actual methods.

Let us explain what happens if $\alpha$ grows. Remember that the spectrum of the harmonic oscillator $-\partial_{x}^{2}+x^{2}$ equals $2 \mathbb{N}+1$ and so is explicit. To our knowledge, that is not the case for the superquadratic oscillator $-\partial_{x}^{2}+x^{2 \alpha}$ if $\alpha$ is greater or equal to 2. That is why we make use of Helffert-Robert's results [HR82] and hence we can study precisely the differences of two different eigenvalues of $\sqrt{-\partial_{x}^{2}+x^{2 \alpha}}$. Indeed, the spectrum of $\sqrt{-\partial_{x}^{2}+x^{2 \alpha}}$ behaves essentially as the sequence $\left(k^{\frac{\alpha}{\alpha+1}}\right)_{k \geq 1}$ and thus is not separated (because $\frac{\alpha}{\alpha+1}<1$ holds). Nevertheless, differences of two different eigenvalues become more and more important as $\alpha$ tends to infinity. This is the key point which allows to reach $A=2$ if $\alpha$ is enough large.

Before stating precisely our results, let us say that analogue Schrödinger equations of (1) are much better understood because the spectrum of $-\partial_{x}^{2}+x^{2 \alpha}$ is separated. Indeed the almost global existence for the Schrödinger equation with quantum harmonic oscillator [GIP09, Ime12a] and superquadratic oscillator $[$ Ime12b] are proved in the nonresonant regime. Here is the definition of solution we will use.

DEFINITION 1.1. - Let $s$ be a positive real number, we say that $w(t, x)$ is a $\mathcal{H}_{\alpha}^{s+1} \times \mathcal{H}_{\alpha}^{s}$ strong solution of (1) on a time interval $I \subset \mathbb{R}$ with initial datum 
$\left(w_{0}, w_{1}\right) \in \mathcal{H}_{\alpha}^{s+1} \times \mathcal{H}_{\alpha}^{s}$ if $w \in \mathcal{C}^{0}\left(I, \mathcal{H}_{\alpha}^{s+1}\right) \cap \mathcal{C}^{1}\left(I, \mathcal{H}_{\alpha}^{s}\right)$ satisfies (1) in the sense of the Duhamel formula for all $t \in I$ :

$$
w(t, \cdot)=\cos \left(t \Lambda_{\alpha, m}\right) w_{0}+\frac{\sin \left(t \Lambda_{\alpha, m}\right)}{\Lambda_{\alpha, m}} w_{1}+\int_{0}^{t} \frac{\sin \left((t-\tau) \Lambda_{\alpha, m}\right) w(\tau, \cdot)^{n+1}}{\Lambda_{\alpha, m}} d \tau
$$

where $\Lambda_{\alpha, m}:=\sqrt{-\partial_{x}^{2}+x^{2 \alpha}+m^{2}}$.

In the sequel $\mathcal{H}_{\alpha}^{s}(\mathbb{R}, \mathbb{R})$ is the subspace of $\mathcal{H}_{\alpha}^{s}$ of functions which take real values.

TheOrem 1.2. - Let $\alpha$ and $n$ be two integers such that $\alpha \geq 4$ and $n \geq 2$. For almost every mass $m>0$, for any $s \gg 1$ and $\left(w_{0}, w_{1}\right) \in \mathcal{H}_{\alpha}^{s+1}(\mathbb{R}, \mathbb{R}) \times$ $\mathcal{H}_{\alpha}^{s}(\mathbb{R}, \mathbb{R})$, there are $C, K \geq 1$ such that if $\varepsilon \ll 1$ holds then (1) admits a unique $\mathcal{H}_{\alpha}^{s+1} \times \mathcal{H}_{\alpha}^{s}$ strong solution with initial datum $(w(0, \cdot), \dot{w}(0, \cdot))=\left(\varepsilon w_{0}, \varepsilon w_{1}\right)$ on the time interval $\left[-C \varepsilon^{-2 n},+C \varepsilon^{-2 n}\right]$. Furthermore one has

$$
|t| \leq C \varepsilon^{-2 n} \quad \Rightarrow \quad\|w(t, \cdot)\|_{\mathcal{H}_{\alpha}^{s+1}}+\|\dot{w}(t, \cdot)\|_{\mathcal{H}_{\alpha}^{s}} \leq K \varepsilon .
$$

THEOREM 1.3. - We suppose the same assumptions that in Theorem 1.2 except that $\alpha$ equals 2 or 3 . If $\alpha=2$ holds, the local existence time can be improved to $\varepsilon^{-n\left(\frac{7}{4}-\rho\right)}$ for any $\rho>0$. If $\alpha=3$ holds, the local existence time can be improved to $\varepsilon^{-n(2-\rho)}$ for any $\rho>0$.

REMARK 1.4. - As said above, it is reasonable to expect that the local existence time can be improved to $\varepsilon^{-n A(\alpha)}$ where $\lim _{\alpha \rightarrow+\infty} A(\alpha)=+\infty$.

REMARK 1.5. - The method allows to prove the same result if the nonlinearity $w^{n+1}$ is replaced by $F(w, x)$ such that $\|F(w, \cdot)\|_{\mathcal{H}_{\alpha}^{s}} \leq C\|w\|_{\mathcal{H}_{\alpha}^{s}}^{n+1}$ holds for $s \gg 1$.

Proofs of Theorem 1.2 and Theorem 1.3 will use multilinear estimates of eigenfunctions which are present in [Ime12b] and a slight modification of estimates of small divisors which are present in [Zha10] (see below Proposition $2.7)$.

For convenience, the integers $n$ and $\alpha$ may not appear in constants.

1.2. Description of the strategy. - Let us order the spectrum of $\Lambda_{\alpha, m}=$ $\sqrt{-\partial_{x}^{2}+x^{2 \alpha}+m^{2}}$ as an increasing sequence :

$$
\begin{gathered}
\operatorname{Sp}\left(\Lambda_{\alpha, 0}\right)=\left\{\lambda_{\alpha, 1}<\lambda_{\alpha, 2}<\cdots\right\}, \quad \lambda_{\alpha, 1}>0, \\
\forall k \geq 1 \quad \Lambda_{\alpha, m} \phi_{\alpha, k}=\left(\lambda_{\alpha, k}^{2}+m^{2}\right)^{\frac{1}{2}} \phi_{\alpha, k} \quad\left\|\phi_{\alpha, k}\right\|_{L^{2}}=1 .
\end{gathered}
$$

We will give precise spectral data in the section 2 . The equation (1) becomes

$$
\begin{aligned}
\left(\partial_{t}^{2}+\Lambda_{\alpha, m}^{2}\right) w & =w^{n+1} \\
\left(i \partial_{t}+\Lambda_{\alpha, m}\right)\left(-i \partial_{t}+\Lambda_{\alpha, m}\right) w & =w^{n+1} .
\end{aligned}
$$


Introduce now $u=:\left(-i \partial_{t}+\Lambda_{\alpha, m}\right) w$. The equality $w=\Lambda_{\alpha, m}^{-1}\left(\frac{u+\bar{u}}{2}\right)$ holds because $w$ takes real values. Hence, $u$ satisfies the following equation

$$
\left(i \partial_{t} u+\Lambda_{\alpha, m}\right) u=F(u), \quad F(u):=\left[\Lambda_{\alpha, m}^{-1}\left(\frac{u+\bar{u}}{2}\right)\right]^{n+1} .
$$

It is easy to check that if $w \in \mathcal{C}^{0}\left(I, \mathcal{H}_{\alpha}^{s+1}(\mathbb{R}, \mathbb{R})\right) \cap \mathcal{C}^{1}\left(I, \mathcal{H}_{\alpha}^{s}(\mathbb{R}, \mathbb{R})\right)$ satisfies (1) in the sense of the Duhamel formula $(2)$, then one has

$\dot{w}(t, \cdot)=-\sin \left(t \Lambda_{\alpha, m}\right) \Lambda_{\alpha, m} w_{0}+\cos \left(t \Lambda_{\alpha, m}\right) w_{1}+\int_{0}^{t} \cos \left((t-\tau) \Lambda_{\alpha, m}\right) w(\tau, \cdot)^{n+1} d \tau$.

Remark also the following fact

$$
\left\|\Lambda_{\alpha, m}^{s} u\right\|_{L^{2}} \simeq\|u\|_{\mathcal{H}_{\alpha}^{s}} \simeq\left\|\partial_{t} w\right\|_{\mathcal{H}_{\alpha}^{s}}+\|w\|_{\mathcal{H}_{\alpha}^{s+1}} .
$$

It is not hard to deduce from previous informations on $u$ that Theorem 1.2 is indeed equivalent to the following result

THEOREM 1.6. - Let $\alpha$ and $n$ be two integers such that $\alpha \geq 4$ and $n \geq 2$. For almost every mass $m>0$, for any $s \gg 1$ and $u_{0} \in \mathcal{H}_{\alpha}^{s}$, there are $C, K \geq 1$ such that if $\varepsilon \ll 1$ holds then (4) admits a unique $\mathcal{H}_{\alpha}^{s}$ strong solution with initial data $u(0)=\varepsilon u_{0}$ on the time interval $\left[-C \varepsilon^{-2 n},+C \varepsilon^{-2 n}\right]$. In other words, $u \in \mathcal{C}^{0}\left(\left[-C \varepsilon^{-2 n},+C \varepsilon^{-2 n}\right], \mathcal{H}_{\alpha}^{s}\right)$ satisfies the Duhamel formula

$$
u(t, \cdot)=\exp \left(i t \Lambda_{\alpha, m}\right) u_{0}-i \int_{0}^{t} \exp \left(i(t-\tau) \Lambda_{\alpha, m}\right) F(u(\tau, \cdot)) d \tau .
$$

Furthermore, one has

$$
|t| \leq C \varepsilon^{-2 n} \quad \Rightarrow \quad\|u(t, \cdot)\|_{\mathcal{H}_{\alpha}^{s}} \leq K \varepsilon
$$

Obviously a similar reformulation of Theorem 1.3 is possible. Theorem 1.6 means that we have to study Sobolev norms of $u$ instead of those of $w$ and $\dot{w}$.

If $s>\frac{1}{2}$ and $\left\|u_{0}\right\|_{\mathcal{H}_{\alpha}^{s}}=\varepsilon \ll 1$, then using a fixed point theorem with the Duhamel formula (5) allows us to get uniqueness of the solution and to prove the local existence time for $u$ is at least of order $\varepsilon^{-n}$. In view to improve the local existence time, let us introduce

$$
\Theta_{s}(u):=\frac{1}{2}\left\|\Lambda_{\alpha, m}^{s} u\right\|_{L^{2}}^{2} .
$$

Remembering (3), one has $\dot{u}=-i w^{n+1}+i \Lambda_{\alpha, m} u$ and a formal derivation of $u$ gives

$$
\begin{aligned}
\frac{d}{d t} \Theta_{s}(u(t, \cdot)) & =\operatorname{Re}\left\langle\Lambda_{\alpha, m}^{s} \dot{u}, \Lambda_{\alpha, m}^{s} u\right\rangle \\
& =\operatorname{Re} i\left\langle\Lambda_{\alpha, m}^{s+1} u, \Lambda_{\alpha, m}^{s} u,\right\rangle-\operatorname{Re} i\left\langle\Lambda_{\alpha, m}^{s} w^{n+1}, \Lambda_{\alpha, m}^{s} u\right\rangle \\
& =\operatorname{Re} i\left\langle\Lambda_{\alpha, m}^{s+\frac{1}{2}} u, \Lambda_{\alpha, m}^{s+\frac{1}{2}} u,\right\rangle-\operatorname{Re} i\left\langle\Lambda_{\alpha, m}^{s} w^{n+1}, \Lambda_{\alpha, m}^{s} u\right\rangle \\
& =-\operatorname{Re} i\left\langle\Lambda_{\alpha, m}^{s} w^{n+1}, \Lambda_{\alpha, m}^{s} u\right\rangle .
\end{aligned}
$$


Indeed, it is possible to make rigorous the previous argument. The Duhamel formula (5) and the fact that $e^{i t \Lambda_{\alpha, m}}$ is an isometry of $\mathcal{H}_{\alpha}^{s}$ imply that

$$
\begin{aligned}
\Theta_{s}(u(t, \cdot))-\Theta_{s}(u(0, \cdot))= & \operatorname{Re} i\left\langle\Lambda_{\alpha, m}^{s} u(0, \cdot), \Lambda_{\alpha, m}^{s} \int_{0}^{t} e^{-i \tau \Lambda_{\alpha, m}} w(\tau, \cdot)^{n+1} d \tau\right\rangle \\
& +\Theta_{s}\left(\int_{0}^{t} e^{-i \tau \Lambda_{\alpha, m}} w(\tau, \cdot)^{n+1} d \tau\right) \\
= & \operatorname{Re} i \int_{0}^{t}\left\langle\Lambda_{\alpha, m}^{s} u(0, \cdot), \Lambda_{\alpha, m}^{s} e^{-i \tau \Lambda_{\alpha, m}} w(\tau, \cdot)^{n+1}\right\rangle d \tau \\
& +\Theta_{s}\left(\int_{0}^{t} e^{-i \tau \Lambda_{\alpha, m}} w(\tau, \cdot)^{n+1} d \tau\right) .
\end{aligned}
$$

Using a second time the Duhamel formula, the previous sum equals

$$
\begin{gathered}
\operatorname{Re} i \int_{0}^{t}\left\langle\Lambda_{\alpha, m}^{s} e^{-i \tau \Lambda_{\alpha, m}} u(\tau, \cdot), \Lambda_{\alpha, m}^{s} e^{-i \tau \Lambda_{\alpha, m}} w(\tau, \cdot)^{n+1}\right\rangle d \tau \\
+\Theta_{s}\left(\int_{0}^{t} e^{-i \tau \Lambda_{\alpha, m}} w(\tau, \cdot)^{n+1} d \tau\right) \\
-\operatorname{Re} \iint_{A(t)}\left\langle\Lambda_{\alpha, m}^{s} e^{-i \tau^{\prime} \Lambda_{\alpha, m}} w\left(\tau^{\prime}, \cdot\right)^{n+1}, \Lambda_{\alpha, m}^{s} e^{-i \tau \Lambda_{\alpha, m}} w(\tau, \cdot)^{n+1}\right\rangle d \tau^{\prime} d \tau
\end{gathered}
$$

where $A(t)$ is the triangle $\left\{\left(\tau, \tau^{\prime}\right) \in[0, t]^{2}, \quad 0 \leq \tau^{\prime} \leq \tau\right\}$. The symmetry $\tau \leftrightarrow$ $\tau^{\prime}$ ensures that the integration on the above triangle is the half integration on the square $[0, t]^{2}$ and thus we can claim

$$
\begin{gathered}
\Theta_{s}(u(t))-\Theta_{s}(u(0))=\operatorname{Re} i \int_{0}^{t}\left\langle\Lambda_{\alpha, m}^{s} u(\tau, \cdot), \Lambda_{\alpha, m}^{s} w(\tau, \cdot)^{n+1}\right\rangle d \tau \\
\frac{d}{d t} \Theta_{s}(u(t, \cdot))=-\operatorname{Re} i\left\langle\Lambda_{\alpha, m}^{s} w^{n+1}, \Lambda_{\alpha, m}^{s} u\right\rangle .
\end{gathered}
$$

Since $w=\Lambda_{\alpha, m}^{-1}\left(\frac{u+\bar{u}}{2}\right), w$ and $u$ contribute essentially at the same degree in all expressions. Therefore $\frac{d}{d t} \Theta_{s}(u)$ can be seen as $\mathbb{R}$-multilinear expression with respect to the variable $u$ of degree $n+2$.

Before stating the main idea, let us say the local existence time $\varepsilon^{-n}$ can be seen as a direct consequence of the following inequality

$$
\left|\frac{d}{d t} \Theta_{s}(u)\right| \leq\|u\|_{\mathcal{H}_{\alpha}^{s}}\left\|w^{n+1}\right\|_{\mathcal{H}_{\alpha}^{s}} \leq\|u\|_{\mathcal{H}_{\alpha}^{s}}\|w\|_{\mathcal{H}_{\alpha}^{s}}^{n+1} \leq\|u\|_{\mathcal{H}_{\alpha}^{s}}^{n+2} .
$$

If one could increase the exponent $n+2$, then one could improve the local existence time. Indeed, this is roughly the idea of normal form. The strategy consists in finding a continuous and multilinear operator

$$
M_{s}:\left(\mathcal{H}_{\alpha}^{s}\right)^{n+2} \rightarrow \mathbb{C}
$$

such that the term $\frac{d}{d t}\left[\Theta_{s}(u(t))-M_{s}(u, \ldots, u)\right]$ has no homogeneous term of degree $n+2$. A formal computation gives 


$$
\begin{aligned}
\frac{d}{d t} M_{s}(u, \ldots, u) & =\sum_{\text {sym }} M_{s}(\dot{u}, u, \ldots, u) \\
& =\sum_{\text {sym }} M_{s}\left(i \Lambda_{\alpha, m} u, u, \ldots, u\right)+\sum_{\text {sym }} M_{s}\left(-i w^{n+1}, u, \ldots, u\right) .
\end{aligned}
$$

Terms of the form $M_{s}\left(i \Lambda_{\alpha, m} u, u, \ldots, u\right)$ will contribute to kill the $(n+2)$ multilinear expression $\frac{d}{d t} \Theta_{s}(u(t))$ of $u$. Thus using boundedness of $M_{s}$ and noticing that $M_{s}\left(-i w^{n+1}, u, \ldots, u\right)$ is of degree $n+1+n+1=2 n+2$ will lead to an inequality of the form

$$
\left|\frac{d}{d t} \Theta_{s}(u)-\frac{d}{d t} M_{s}(u, \ldots, u)\right| \leq C|| u \|_{\mathcal{H}_{\alpha}^{s}}^{2 n+2} .
$$

See Lemma 5.1 for a rigorous explanation of the previous computation. A new time, the boundedness of $M_{s}$ on $\left(\mathcal{H}_{\alpha}^{s}\right)^{n+2}$ implies that

$$
\left|\Theta_{s}(u(t))-\Theta_{s}(u(0))\right| \leq C t \sup _{0 \leq t^{\prime} \leq t}\left\|u\left(t^{\prime}\right)\right\|_{\mathcal{H}_{\alpha}^{s}}^{2 n+2}+C \sup _{0 \leq t^{\prime} \leq t}\left\|u\left(t^{\prime}\right)\right\|_{\mathcal{H}_{\alpha}^{s}}^{n+2}
$$

If $\|u(0)\|_{\mathcal{H}_{s}^{\alpha}}=\varepsilon$ is enough small, then the inequality $\|u(t)\|_{\mathcal{H}_{s}^{\alpha}} \leq K \varepsilon$ is true provided that

$$
\varepsilon^{2} \leq C t \varepsilon^{2 n+2}+C \varepsilon^{n+2}
$$

$$
\frac{\varepsilon^{2}-C \varepsilon^{n+2}}{C \varepsilon^{2 n+2}} \leq t
$$

In other words $t \geq C \varepsilon^{-2 n}$.

In the papers [Del09, FZ10, Zha10] and Theorem 1.6, such a method fails and the map $M_{s}$ leaves some homogeneous term of degree $n+2$ in $\frac{d}{d t}\left[\Theta_{s}(u(t))-M(u, \ldots, u)\right]$. In the following table, we recap the improved local existence time expected depending on whether we eliminate $(n+2)$ homogeneous terms in $\operatorname{Re} i\left\langle\Lambda_{\alpha, m}^{s} w^{n+1}, \Lambda_{\alpha, m}^{s} u\right\rangle$.

\begin{tabular}{|c|c|c|l|}
\hline elimination & no & partial & total \\
\hline time expected & $\varepsilon^{-n}$ & $\varepsilon^{-A n}$ & $\varepsilon^{-2 n}$ \\
& local existence & $A \in] 1,2[$ & \\
\hline
\end{tabular}

1.3. Construction of $M_{s}$ and small divisors. - In this section we explain why there is only one reasonable way to define the multilinear form $M_{s}$ described previously.

Roughly speaking, a bounded $\mathbb{R}$-multilinear form $M_{s}$ of degree $n+2$ on $\mathcal{H}_{\alpha}^{s}$ is essentially defined by the values of $M\left(\phi_{\alpha, k_{0}}, \ldots, \phi_{\alpha, k_{n+1}}\right)$ because of the expansion

$$
M_{s}\left(u_{0}, \ldots, u_{n+1}\right)=\sum_{\mathbb{N}^{n+2}} M_{s}\left(\phi_{\alpha, k_{0}}, \ldots, \phi_{\alpha, k_{n+1}}\right) \prod_{j=0}^{n+1}\left\langle u_{j}, \phi_{\alpha, k_{j}}\right\rangle .
$$


The expression $\frac{d}{d t} \Theta_{s}(u)$ will essentially involve multilinear integrals

$$
\int_{\mathbb{R}} \phi_{\alpha, k_{0}}(x) \ldots \phi_{\alpha, k_{n+1}}(x) d x
$$

As said in part 1.2 , we want to construct $M_{s}$ such that

$$
\frac{d}{d t} \Theta_{s}(u(t))=M_{s}\left(i \Lambda_{\alpha, m} u, u, \ldots, u\right)+\cdots+M_{s}\left(u, \ldots, u, i \Lambda_{\alpha, m} u\right) .
$$

Using (6), we see there are some $\mathbb{R}$-multilinear form $\Theta_{s, 0}, \ldots, \Theta_{s, n+1}$ such that

$$
\frac{d}{d t} \Theta_{s}(u(t))=\sum_{\ell=0}^{n+1} \Theta_{s, \ell}(\underbrace{\bar{u}, \ldots, \bar{u}}_{\ell}, \underbrace{u, \ldots, u}_{n+2-\ell}) .
$$

Giving the exact definition of $\Theta_{s, \ell}$ is not necessary in this section and will be done in part 4 , we only aim to explain the general idea. Hence, it is natural to look for $M_{s}$ of the same form :

$$
M_{s}(u, \ldots, u)=\sum_{\ell=0}^{n+1} M_{s, \ell}(\underbrace{\bar{u}, \ldots, \bar{u}}_{\ell}, \underbrace{u, \ldots, u}_{n+2-\ell}) .
$$

Therefore, in view to have (8), it suffices to choose $M_{s, \ell}$ such that

$$
\begin{gathered}
\Theta_{s, \ell}(\underbrace{\bar{u}, \ldots, \bar{u}}_{\ell}, \underbrace{u, \ldots, u}_{n+2-\ell})=\sum_{j=1}^{\ell} M_{s, \ell}(\underbrace{\bar{u}, \ldots, \bar{u}}_{j-1},-i \Lambda_{\alpha, m} \bar{u}, \underbrace{\bar{u}, \ldots, \bar{u}}_{\ell-j}, \underbrace{u, \ldots, u}_{n+2-\ell}) \\
+\sum_{j=\ell+1}^{n+2} M_{s, \ell}(\underbrace{\bar{u}, \ldots, \bar{u}}_{\ell}, \underbrace{u, \ldots, u}_{j-\ell-1}, i \Lambda_{\alpha, m} u, \underbrace{u, \ldots, u}_{n+2-j}) .
\end{gathered}
$$

Notice that the equality $\Lambda_{\alpha, m} \bar{u}=\overline{\Lambda_{\alpha, m} u}$ holds because $\Lambda_{\alpha, m}$ has a real spectrum and real eigenfunctions. Now, we consider those forms on the eigenbasis $\left(\phi_{\alpha, k}\right)_{k \geq 1}$ and see that $\Theta_{s, \ell}\left(\phi_{\alpha, k_{0}}, \ldots, \phi_{\alpha, k_{n+1}}\right)$ equals

$$
-i\left[\sum_{j=0}^{n+1}\left(\mathbf{1}_{j \leq \ell}-\mathbf{1}_{j>\ell}\right) \sqrt{\lambda_{\alpha, k_{j}}^{2}+m^{2}}\right] M_{s, \ell}\left(\phi_{\alpha, k_{0}}, \ldots, \phi_{\alpha, k_{n+1}}\right) .
$$

The last expression means that once we have $\frac{d}{d t} \Theta_{s}$, there is one way to define $M_{s, \ell}$ :

$$
M_{s, \ell}\left(\phi_{\alpha, k_{0}}, \ldots, \phi_{\alpha, k_{n+1}}\right)=\frac{i}{\sum_{j=0}^{n+1} \pm \sqrt{\lambda_{\alpha, k_{j}}^{2}+m^{2}}} \Theta_{s, \ell}\left(\phi_{\alpha, k_{0}}, \ldots, \phi_{\alpha, k_{n+1}}\right) .
$$

Now remember that $\frac{d}{d t} \Theta_{s}(u)$ is a continuous function of $u \in \mathcal{H}_{\alpha}^{s}$ for $s>\frac{1}{2}$ (see the line $(7))$. So are $\Theta_{s, \ell}$. In the following we will write a model of multilinear forms $M_{s}$ such that some estimates on the values $M_{s}\left(\phi_{\alpha, k_{0}}, \ldots, \phi_{\alpha, k_{n+1}}\right)$ imply 
the boundedness on $\left(\mathcal{H}_{\alpha}^{s}\right)^{2 n+2}$. For this, we will need to bound from below linear combinations of eigenvalues of $\Lambda_{\alpha, m}$ such that the definition (9) will not lose regularity. Indeed, our estimates are directly adapted from [Zha10] and are available for almost every $m>0$. This issue is usually called a "small divisor problem".

\section{Spectral analysis}

2.1. Suitable Sobolev spaces and eigenvalues. - We recall some well known properties fulfilled by $\Lambda_{\alpha, 0}=\sqrt{-\partial_{x}^{2}+x^{2 \alpha}}$ and lots of usual pseudodifferential operators which act in a one-dimensional space (see for instance [BS91, Chapter 2.3]).

Proposition 2.1. - The operator $\Lambda_{\alpha, 0}: \mathcal{S}(\mathbb{R}) \rightarrow \mathcal{S}(\mathbb{R})$ is essentially selfadjoint. Its spectrum is an increasing and positive sequence $\left(\lambda_{\alpha, k}\right)_{k \geq 1}$ which tends to $+\infty$. Moreover, there is an orthonormal basis $\left(\phi_{\alpha, k}\right)_{k \geq 1}$ of $L^{2}(\mathbb{R})$ such that for all $k \geq 1$ one has

a) $\overline{\phi_{\alpha, k}}=\phi_{\alpha, k}$

b) $\phi_{\alpha, k} \in \mathcal{S}(\mathbb{R})$

c) the eigenspace $\operatorname{ker}\left(\Lambda_{\alpha, 0}-\lambda_{\alpha, k} I\right) \cap L^{2}(\mathbb{R})$ is spanned by $\phi_{\alpha, k}$.

For the next proposition, see for instance [YZ04, Lemma 2.4].

Proposition 2.2. - Given a number $s>0$ and a distribution $f \in \mathcal{S}^{\prime}(\mathbb{R})$, the following assertions are equivalent

i) $f \in \operatorname{Dom}\left(\Lambda_{\alpha, 0}^{s}\right)$, or equivalently $\Lambda_{\alpha, 0} f \in L^{2}(\mathbb{R})$

ii) $f$ admits a decomposition $f=\sum_{k \geq 1} z_{k} \phi_{\alpha, k}$ with $\sum_{k \geq 1}\left|z_{k}\right|^{2} \lambda_{\alpha, k}^{2 s}<+\infty$.

iii) $f \in H^{s}(\mathbb{R})$ and $x \mapsto x^{\alpha s} f(x) \in \bar{L}^{2}(\mathbb{R})$

The vector space of such $f$ is defined to be $\mathcal{H}_{\alpha}^{s}$ and admits one of any equivalent norm :

$$
\|f\|_{H^{s}}+\left\|x^{\alpha s} f(x)\right\|_{L^{2}(\mathbb{R})} \simeq\left(\sum_{k \geq 1}\left|z_{k}\right|^{2} \lambda_{\alpha, k}^{2 s}<+\infty\right)^{\frac{1}{2}} .
$$

2.2. Helffer-Robert's asymptotic expansion. - In the sequel, we will write $\alpha^{\prime}=\frac{\alpha}{\alpha+1}$. The Weyl formula (see [RS78, Theorem XIII.81]) states

$$
N(E):=\operatorname{Card}\left(k \geq 1, \lambda_{\alpha, k}^{2} \leq E\right) \simeq \frac{2}{2 \pi} \int_{\left\{x, x^{2 \alpha} \leq E\right\}} \sqrt{E-x^{2 \alpha}} d x \simeq c E^{1 / 2 \alpha^{\prime}} .
$$

As eigenvalues $\lambda_{\alpha, k}$ are simple, one deduces the following asymptotic

$$
\lambda_{\alpha, k} \simeq k^{\alpha^{\prime}} .
$$


Therefore, a convenient norm of the space $\mathcal{H}_{\alpha}^{s}$ is also

$$
\left\|\sum_{k \geq 1} z_{k} \phi_{\alpha, k}\right\|_{\mathcal{H}_{\alpha}^{s}}=\left(\sum_{k \geq 1}\left|z_{k}\right|^{2} k^{2 \alpha^{\prime} s}\right)^{\frac{1}{2}}<+\infty .
$$

For our purpose, (10) will not be sufficient. It turns out that a more accurate asymptotic expansion of $\left(\lambda_{\alpha, k}\right)_{k \geq 1}$ is true :

$$
\lambda_{\alpha, k}=\sum_{\ell=0}^{\alpha+1} c_{\ell}(\alpha) k^{\frac{\alpha-\ell}{\alpha+1}}+\mathcal{O}\left(\frac{1}{k^{\frac{2}{\alpha+1}}}\right), \quad c_{0}(\alpha)>0 .
$$

The previous expansion is a consequence of the following result [HR82, theorem (2-2), page 858].

TheOrem 2.3. - Consider $\alpha, \beta \in \mathbb{N}^{\star}$ and $V$ a real polynomial of degree $2 \alpha$ such that $\lim _{ \pm \infty} V(x)=+\infty$ holds. If $\left(\mu_{k}\right)_{k \geq 1}$ is the sequence of eigenvalues of $-\partial_{x}^{2 \beta}+V(x)$ on $L^{2}(\mathbb{R})$, then there is a sequence $\left(b_{k}\right)_{k \geq 0}$ and a real number $\sigma>0$ such that $b_{0}>0$ and

$$
\mu_{k}^{(\alpha+\beta) /(2 \alpha \beta)} \simeq(k+\sigma) \sum_{\ell \geq 0} b_{\ell}(k+\sigma)^{-\ell /(\alpha+\beta)}
$$

The following result gives a crucial separation property fulfilled by the sequence $\left(\lambda_{\alpha, k}\right)_{k \geq 1}$.

Proposition 2.4. - There is a constant $C>1$, which may depend on $\alpha$, such that

$$
\forall k_{1}>k_{2} \geq 1 \quad \frac{1}{C}\left(k_{1}^{\alpha^{\prime}}-k_{2}^{\alpha^{\prime}}\right) \leq \lambda_{\alpha, k_{1}}-\lambda_{\alpha, k_{2}} \leq C\left(k_{1}^{\alpha^{\prime}}-k_{2}^{\alpha^{\prime}}\right) .
$$

Proof. - For any integers $k_{1}$ and $k_{2}$ such that $k_{1}>k_{2} \geq 1$, one has

$$
\begin{aligned}
\frac{1}{k_{2}^{\frac{2}{\alpha+1}}} & =\frac{\left(k_{1}^{\frac{\alpha}{\alpha+1}}-k_{2}^{\frac{\alpha}{\alpha+1}}\right)}{k_{2}^{\frac{2}{\alpha+1}}\left(k_{1}^{\frac{\alpha}{\alpha+1}}-k_{2}^{\frac{\alpha}{\alpha+1}}\right)} \\
& \leq\left(k_{1}^{\frac{\alpha}{\alpha+1}}-k_{2}^{\frac{\alpha}{\alpha+1}}\right)\left(\frac{1}{k_{1}^{\frac{\alpha}{\alpha+1}}-1}+\frac{1}{\left(k_{1}-1\right)^{\frac{2}{\alpha+1}}\left(k_{1}^{\frac{\alpha}{\alpha+1}}-\left(k_{1}-1\right)^{\frac{\alpha}{\alpha+1}}\right)}\right) .
\end{aligned}
$$

Hence, we can write

$$
\frac{1}{k_{1}^{\frac{2}{\alpha+1}}}+\frac{1}{k_{2}^{\frac{2}{\alpha+1}}} \leq \frac{2}{k_{2}^{\frac{2}{\alpha+1}}} \leq\left(k_{1}^{\frac{\alpha}{\alpha+1}}-k_{2}^{\frac{\alpha}{\alpha+1}}\right) o\left(k_{1}\right) .
$$

Now remember the concavity inequality :

$$
\forall \omega \in] 0,1\left[\quad \forall y>x>0 \quad \frac{\omega}{y^{1-\omega}} \leq \frac{y^{\omega}-x^{\omega}}{y-x} \leq \frac{1}{y^{1-\omega}} .\right.
$$


One deduces

$$
\forall \ell>0 \quad\left|k_{1}^{\frac{\alpha-\ell}{\alpha+1}}-k_{2}^{\frac{\alpha-\ell}{\alpha+1}}\right| \leq\left(k_{1}^{\frac{\alpha}{\alpha+1}}-k_{2}^{\frac{\alpha}{\alpha+1}}\right) o\left(k_{1}\right)
$$

The above inequalities (12) and (14), combined with (11), ensure that $k_{1}^{\frac{\alpha}{\alpha+1}}-$ $k_{2}^{\frac{\alpha}{\alpha+1}}$ and $\lambda_{\alpha, k_{1}}-\lambda_{\alpha, k_{2}}$ are equivalent as $k_{1}=\max \left(k_{1}, k_{2}\right)$ tends to infinity. Proposition 2.4 is proved.

2.3. Multilinear integrals of eigenfunctions. - Let us recall estimates from [Ime12b, Proposition 2.5.1].

Proposition 2.5. - For all integers $\alpha \geq 1, n \geq 2, N \geq 1$, there are $C=C(\alpha, n, N)>0$ and $\nu=\nu(\alpha, n)>0$ such that for all $\left(k_{0}, \ldots, k_{n+1}\right) \in$ $(\mathbb{N} \backslash\{0\})^{n+2}$ we have

$$
\int_{\mathbb{R}}\left|\phi_{\alpha, k_{0}}(x) \ldots \phi_{\alpha, k_{n+1}}(x) d x\right| \leq C k_{3}^{\star \alpha^{\prime} \nu}\left(\frac{k_{2}^{\star \alpha^{\prime}} k_{3}^{\star \alpha^{\prime}}}{k_{2}^{\star \alpha^{\prime}} k_{3}^{\star \alpha^{\prime}}+k_{1}^{\star 2 \alpha^{\prime}}-k_{2}^{\star 2 \alpha^{\prime}}}\right)^{N}
$$

where $k_{1}^{\star} \geq k_{2}^{\star} \geq k_{3}^{\star}$ are the largest integers among $k_{0}, \ldots, k_{n+1}$.

For our purpose, we will need weaker estimates.

Corollary 2.6. - Let $\delta$ be an arbitrary number in $] 0,1\left[\right.$ and $\left(k_{0}, \ldots, k_{n+1}\right) \in$ $(\mathbb{N} \backslash\{0\})^{n+2}$ such that $\max \left(k_{1}, \ldots, k_{n}\right) \leq k_{n+1}$. The following two assertions hold

i) in the zone $\frac{k_{0}}{k_{n+1}} \in\left[\delta, \frac{1}{\delta}\right]$ and $\max \left(k_{1}, \ldots, k_{n}\right) \leq k_{n+1}$, one can replace the right member of (15) by

$$
C \max \left(k_{1}^{\alpha^{\prime}}, \ldots, k_{n}^{\alpha^{\prime}}\right)^{\nu}\left(\frac{\max \left(k_{1}^{\alpha^{\prime}}, \ldots, k_{n}^{\alpha^{\prime}}\right)}{\max \left(k_{1}^{\alpha^{\prime}}, \ldots, k_{n}^{\alpha^{\prime}}\right)+\left|k_{0}^{\alpha^{\prime}}-k_{n+1}^{\alpha^{\prime}}\right|}\right)^{N},
$$

ii) if $\frac{k_{0}}{k_{n+1}} \notin\left[\delta, \frac{1}{\delta}\right]$ or $\max \left(k_{1}, \ldots, k_{n}\right)>\delta^{2} k_{n+1}$, one can replace the right member of (15) by

$$
C \frac{\max _{2}\left(k_{1}^{\alpha^{\prime}}, \ldots, k_{n+1}^{\alpha^{\prime}}\right)^{\nu+N}}{\left(k_{0}^{\alpha^{\prime}}+\cdots+k_{n+1}^{\alpha^{\prime}}\right)^{N}} .
$$

Proof. - i) Without loss of generality, we may suppose $k_{0} \geq k_{n+1}$. Using notations $k_{1}^{\star}, k_{2}^{\star}, k_{3}^{\star}$ as in (15), it is clear that

$$
k_{1}^{\star}=k_{0}, \quad k_{2}^{\star}=k_{n+1}, \quad k_{3}^{\star}=\max \left(k_{1}, \ldots, k_{n}\right) .
$$

Thus

$$
\left|k_{1}^{\star 2 \alpha^{\prime}}-k_{2}^{\star 2 \alpha^{\prime}}\right|=\left|k_{1}^{\star \alpha^{\prime}}-k_{2}^{\star \alpha^{\prime}}\right|\left|k_{1}^{\star \alpha^{\prime}}+k_{2}^{\star \alpha^{\prime}}\right| \geq\left|k_{0}^{\alpha^{\prime}}-k_{n+1}^{\alpha^{\prime}}\right| k_{n+1}^{\alpha^{\prime}} .
$$

Finally, Point i) comes from (15). 
ii) We consider several subcases :

iia) If $k_{0} \leq k_{n+1}$ and $\max \left(k_{1}, \ldots, k_{n}\right) \leq \delta^{2} k_{n+1}$. We have $\delta^{-1} k_{0} \leq k_{n+1}$ and thus

$$
\begin{aligned}
\left|k_{1}^{\star} 2 \alpha^{\prime}-k_{2}^{\star 2 \alpha^{\prime}}\right| & \geq C_{\delta} k_{n+1}^{2 \alpha^{\prime}} \geq C_{\delta, n} k_{n+1}^{\alpha^{\prime}}\left(k_{0}^{\alpha^{\prime}}+\cdots+k_{n+1}^{\alpha^{\prime}}\right) \\
k_{2}^{\star \alpha^{\prime}} k_{3}^{\star \alpha^{\prime}} & \leq k_{n+1}^{\alpha^{\prime}} \max _{2}\left(k_{1}^{\alpha^{\prime}}, \ldots, k_{n+1}^{\alpha^{\prime}}\right) .
\end{aligned}
$$

Hence, we can bound (15) to get (16).

iib) If $k_{n+1} \leq k_{0}$ and $\max \left(k_{1}, \ldots, k_{n}\right) \leq \delta^{2} k_{n+1}$. We have $\delta^{-1} k_{n+1} \leq k_{0}$ and

$$
\begin{aligned}
\left|k_{1}^{\star} 2 \alpha^{\prime}-k_{2}^{\star 2 \alpha^{\prime}}\right| & \geq C_{\delta} k_{0}^{2 \alpha^{\prime}} \geq C_{\delta, n} k_{0}^{\alpha^{\prime}}\left(k_{0}^{\alpha^{\prime}}+\cdots+k_{n+1}^{\alpha^{\prime}}\right) \\
k_{2}^{\star \alpha^{\prime}} k_{3}^{\star \alpha^{\prime}} & \leq C_{\delta} k_{0}^{\alpha^{\prime}} \max _{2}\left(k_{1}^{\alpha^{\prime}}, \ldots, k_{n+1}^{\alpha^{\prime}}\right) .
\end{aligned}
$$

A new time, (16) comes from (15).

iic) If $\delta^{2} k_{n+1} \leq \max \left(k_{1}, \ldots, k_{n}\right)$ and $k_{0} \leq 2 k_{n+1}$. Notice that $\max \left(k_{1}, \ldots, k_{n}\right) \leq$ $k_{n+1}$ also holds in the hypothesis of Corollary 2.6, hence we have

$$
\frac{\max _{2}\left(k_{1}^{\alpha^{\prime}}, \ldots, k_{n+1}^{\alpha^{\prime}}\right)}{k_{0}^{\alpha^{\prime}}+\cdots+k_{n+1}^{\alpha^{\prime}}} \geq C_{n, \delta} \frac{\max \left(k_{1}^{\alpha^{\prime}}, \ldots, k_{n}^{\alpha^{\prime}}\right)}{k_{n+1}^{\alpha^{\prime}}} \geq \delta^{2 \alpha^{\prime}} C_{n, \delta} .
$$

To conclude, remark the left side of (15) is clearly bounded by $k_{3}^{\star \alpha^{\prime} \nu} \leq$ $\max _{2}\left(k_{1}, \ldots, k_{n+1}\right)^{\alpha^{\prime} \nu}$.

iid) If $\delta^{2} k_{n+1} \leq \max \left(k_{1}, \ldots, k_{n}\right)$ and $k_{0}>2 k_{n+1}$. We just write

$$
\frac{k_{2}^{\star \alpha^{\prime}} k_{3}^{\star \alpha^{\prime}}}{k_{2}^{\star \alpha^{\prime}} k_{3}^{\star \alpha^{\prime}}+k_{1}^{\star 2 \alpha^{\prime}}-k_{2}^{\star 2 \alpha^{\prime}}} \leq C_{\delta} \frac{k_{n+1}^{2 \alpha^{\prime}}}{k_{0}^{2 \alpha^{\prime}}} \leq C_{\delta} \frac{k_{n+1}^{\alpha^{\prime}}}{k_{0}^{\alpha^{\prime}}} \leq C_{n, \delta} \frac{\max _{2}\left(k_{1}^{\alpha^{\prime}}, \ldots, k_{n+1}^{\alpha^{\prime}}\right)}{k_{0}^{\alpha^{\prime}}+\cdots+k_{n+1}^{\alpha^{\prime}}} .
$$

2.4. Small divisors. - Let us define two maps $F_{m}^{\ell}$ and $\widehat{F}_{m}^{\ell}$ on $\mathbb{R}^{2 n+2}$

$$
\begin{gathered}
F_{m}^{\ell}\left(\xi_{0}, \ldots, \xi_{n+1}\right)=\sum_{j=0}^{\ell} \sqrt{\xi_{j}^{2}+m^{2}}-\sum_{j=\ell+1}^{n+1} \sqrt{\xi_{j}^{2}+m^{2}} \\
\widehat{F}_{m}^{\ell}\left(\xi_{0}, \ldots, \xi_{n+1}\right)=\sum_{j=0}^{\ell} \sqrt{\xi_{j}^{2}+m^{2}}-\sum_{j=\ell+1}^{n} \sqrt{\xi_{j}^{2}+m^{2}}+\sqrt{\xi_{n+1}^{2}+m^{2}} .
\end{gathered}
$$

We also define specific subsets of $(\mathbb{N} \backslash\{0\})^{n+2}$ :

$$
\begin{aligned}
& \Omega_{n+2}(\ell):=\left\{\left(k_{0}, \ldots, k_{n+1}\right), \quad\left\{k_{0}, \ldots, k_{\ell}\right\}=\left\{k_{\ell+1}, \ldots, k_{n+1}\right\}\right\}, \\
& \Omega_{n+2}(\widehat{\ell}):=\left\{\left(k_{0}, \ldots, k_{n+1}\right), \quad\left\{\left(k_{n+1}, k_{0}, \ldots, k_{\ell}\right\}=\left\{k_{\ell+1}, \ldots, k_{n}\right\}\right\} .\right.
\end{aligned}
$$

The following result is proved in [Zha10, Theorem 2.3.1 and Proposition 2.3.6] for $\alpha=1$ (and so $\alpha^{\prime}=\frac{\alpha}{1+\alpha}=\frac{1}{2}$ ). The exponent in (18) equals $3+\rho$ in [Zha10] instead of $1+\frac{2}{\alpha}+\rho$. 
Proposition 2.7. - For all $\rho>0, \ell \in[[0, n+1]]$ and almost every $m>0$ (in the sense of Lebesgue), there are $C$ and $\nu_{0}$ such that for all $\left(k_{0}, \ldots, k_{n+1}\right) \in$ $(\mathbb{N} \backslash\{0\})^{n+2} \backslash \Omega_{n+2}(\ell)$ we have

$$
\frac{1}{\left|F_{m}^{\ell}\left(\lambda_{\alpha, k_{0}}, \ldots, \lambda_{\alpha, k_{n+1}}\right)\right|} \leq \frac{C\left(k_{0}^{\alpha^{\prime}}+k_{n+1}^{\alpha^{\prime}}\right)^{1+\frac{2}{\alpha}+\rho}}{\left[\left|k_{0}^{\alpha^{\prime}}-k_{n+1}^{\alpha^{\prime}}\right|+\max \left(k_{1}^{\alpha^{\prime}}, \ldots, k_{n}^{\alpha^{\prime}}\right)\right]^{-\nu_{0}}}
$$

and

$$
\frac{1}{\left|F_{m}^{\ell}\left(\lambda_{\alpha, k_{0}}, \ldots, \lambda_{\alpha, k_{n+1}}\right)\right|} \leq C\left(k_{1}^{\alpha^{\prime}}+\cdots+k_{n}^{\alpha^{\prime}}\right)^{\nu_{0}}
$$

Moreover, if $\left(k_{0}, \ldots, k_{n+1}\right) \in(\mathbb{N} \backslash\{0\})^{n+2} \backslash \Omega_{n+2}(\widehat{\ell})$ then we have

$$
\begin{aligned}
\frac{1}{\left|\widehat{F}_{m}^{\ell}\left(\lambda_{\alpha, k_{0}}, \ldots, \lambda_{\alpha, k_{n+1}}\right)\right|} \leq C \frac{\left(k_{1}^{\alpha^{\prime}}+\cdots+k_{n}^{\alpha^{\prime}}\right)^{\nu_{0}}}{k_{0}^{\alpha^{\prime}}+k_{n+1}^{\alpha^{\prime}}} \\
\frac{1}{\left|\widehat{F}_{m}^{\ell}\left(\lambda_{\alpha, k_{0}}, \ldots, \lambda_{\alpha, k_{n+1}}\right)\right|} \leq C\left(k_{1}^{\alpha^{\prime}}+\cdots+k_{n}^{\alpha^{\prime}}\right)^{\nu_{0}} .
\end{aligned}
$$

REMARK 2.8. - Since parameters $\alpha$ and $n$ take values in $\mathbb{N}^{2}$, the conclusion of the previous proposition is of course also available for all $(\alpha, n)$.

A similar argumentation proves Proposition 2.7. The main idea is to consider the complementary set of such real numbers $m$ and to bound its Lebesgue measure by arbitrary small numbers. For that point, we need to deal with convergence of some series (see [Zha10, page 659]). Therefore, the only thing to change in [Zha10, page 659] in view to prove Proposition 2.7 is dealing with an adequate series involving the exponent $1+\frac{2}{\alpha}+\rho$ instead of $3+\rho$ in (18). It comes from the following lemma.

Lemma 2.9. - For any $N>1$ and $\rho>0$ one has

$$
\sum_{k_{0}, k_{n+1} \geq 1} \frac{1}{\left(\lambda_{\alpha, k_{0}}+\lambda_{\alpha, k_{n+1}}\right)^{1+\frac{2}{\alpha}+\rho}\left(1+\left|\lambda_{\alpha, k_{0}}-\lambda_{\alpha, k_{n+1}}\right|\right)^{N}}<+\infty .
$$

Before giving the proof of Lemma 2.9, the form of the series (22) explains why it is so important to understand the behavior of the differences $\lambda_{\alpha, k_{0}}-\lambda_{\alpha, k_{n+1}}$ (see Proposition 2.4).

Proof. - Defining $\eta=1+\frac{2}{\alpha}+\rho$, one sees

$$
\alpha^{\prime} \eta-\left(1-\alpha^{\prime}\right)>\frac{\alpha}{1+\alpha}\left(1+\frac{2}{\alpha}\right)-\frac{1}{1+\alpha}=1
$$

It suffices obviously to prove (22) for a real $N$ which is enough closed to 1 . We choose some $N>1$ such that $\alpha^{\prime} \eta-\left(1-\alpha^{\prime}\right) N>1$ holds. For a constant $C$ 
which may depend on $\alpha$, the double series in (22) is less than

$$
\sum_{k_{0}>k_{n+1}} \frac{C}{\left(k_{0}+k_{n+1}\right)^{\eta \alpha^{\prime}}\left(k_{0}^{\alpha^{\prime}}-k_{n+1}^{\alpha^{\prime}}\right)^{N}} \leq \sum_{k_{0}>k_{n+1}} \frac{C k_{0}^{\left(1-\alpha^{\prime}\right) N}}{\left(k_{0}+k_{n+1}\right)^{\eta \alpha^{\prime}}\left(k_{0}-k_{n+1}\right)^{N}} .
$$

Setting $b=k_{0}-k_{n+1} \geq 1$ and $a=k_{0}$, we can bound by

$$
C \sum_{a, b \geq 1} \frac{1}{a^{\alpha^{\prime} \eta-\left(1-\alpha^{\prime}\right) N} b^{N}}=C\left(\sum_{a \geq 1} \frac{1}{a^{\alpha^{\prime} \eta-\left(1-\alpha^{\prime}\right) N}}\right)\left(\sum_{b \geq 1} \frac{1}{b^{N}}\right)<+\infty .
$$

REMARK 2.10. - Unfortunately, the condition $\eta>1+\frac{2}{\alpha}$ is optimal to get (22). In fact, we can bound from below the series in (22) by

$$
C \sum_{\aleph} \frac{1}{\left(k_{0}+k_{n+1}\right)^{\eta \alpha^{\prime}}} \geq C \sum_{k_{n+1}=1}^{+\infty} \frac{k_{n+1}^{1-\alpha^{\prime}}}{\left(2 k_{n+1}+k_{n+1}^{1-\alpha^{\prime}}\right)^{\eta \alpha^{\prime}}} \simeq \sum_{k_{n+1}=1}^{\infty} \frac{1}{k_{n+1}^{\eta \alpha^{\prime}-1+\alpha^{\prime}}} .
$$

where $\aleph=\left\{k_{n+1}<k_{0}<k_{n+1}+k_{n+1}^{1-\alpha^{\prime}}\right\}$. It turns out the condition $\eta \alpha^{\prime}-1+\alpha^{\prime}>$ 1 is the same that $\eta>1+\frac{2}{\alpha}$.

\section{Multilinear models}

3.1. A first class of multilinear operators. - Let $\mathcal{S}(\mathbb{R})$ and $\mathcal{S}^{\prime}(\mathbb{R})$ denote, respectively, the usual Schwartz class and and the space of tempered distributions. Instead of defining a multilinear operator $M: \mathcal{S}(\mathbb{R})^{n+2}(\mathbb{R}) \rightarrow \mathbb{C}$, we will a define multilinear operator $\mathcal{M}: \mathcal{S}(\mathbb{R})^{n} \rightarrow \mathcal{L}\left(\mathcal{S}(\mathbb{R}), \mathcal{S}^{\prime}(\mathbb{R})\right)$. Remembering that functions in Sobolev spaces $\mathcal{H}_{\alpha}^{s}$ have a unique decomposition in the eigenbasis $\left(\phi_{\alpha, k}\right)_{k \geq 1}$, it is natural to consider

$$
\left\langle\mathcal{M}\left(\phi_{\alpha, k_{1}}, \ldots, \phi_{\alpha, k_{n}}\right) \phi_{\alpha, k_{n+1}}, \phi_{\alpha, k_{0}}\right\rangle \text {. }
$$

It turns out that the class of operators $\mathcal{M}$ we will consider are defined by suitable estimates of terms (23).

Definition 3.1. - Let $\tau, \nu, \delta$ be positive real numbers and $n$ be a positive integer. A multilinear operator $\mathcal{M}: \mathcal{S}(\mathbb{R})^{n} \rightarrow \mathcal{L}\left(\mathcal{S}(\mathbb{R}), \mathcal{S}^{\prime}(\mathbb{R})\right)$ is in the class $\mathcal{M}_{\alpha, n, \delta}^{\tau, \nu}$ if the following conditions hold for all $N>0$

i) if $\delta \leq \frac{k_{n+1}}{k_{0}} \leq \frac{1}{\delta}$ and $\max \left(k_{1}, \ldots, k_{n}\right) \leq k_{n+1}$ then

$$
\left|\left\langle\mathcal{M}\left(\phi_{\alpha, k_{1}}, \ldots, \phi_{\alpha, k_{n}}\right) \phi_{\alpha, k_{n+1}}, \phi_{\alpha, k_{0}}\right\rangle\right| \leq \frac{C k_{0}^{\alpha^{\prime} \tau} \max \left(k_{1}^{\alpha^{\prime}}, \ldots, k_{n}^{\alpha^{\prime}}\right)^{\nu+N}}{\left(\max \left(k_{1}^{\alpha^{\prime}}, \ldots, k_{n}^{\alpha^{\prime}}\right)+\left|k_{0}^{\alpha^{\prime}}-k_{n+1}^{\alpha^{\prime}}\right|\right)^{N}}
$$

ii) for all others $\left(k_{0}, \ldots, k_{n+1}\right)$, the term $\left\langle\mathcal{M}\left(\phi_{\alpha, k_{1}}, \ldots, \phi_{\alpha, k_{n}}\right) \phi_{\alpha, k_{n+1}}, \phi_{\alpha, k_{0}}\right\rangle$ is zero. 
The previous definition means essentially that an operator $\mathcal{M} \in \mathcal{M}_{\alpha, n, \delta}^{\tau, \nu}$ can be expressed as the following :

$$
\left\langle\mathcal{M}\left(u_{1}, \ldots, u_{n}\right) u_{n+1}, u_{0}\right\rangle=\sum_{(\mathbb{N} \backslash\{0\})^{n+2}} m\left(k_{0}, \ldots, k_{n+1}\right) \prod_{j=0}^{n+1}\left\langle u_{j}, \phi_{\alpha, k_{j}}\right\rangle
$$

where the map $\left(k_{0}, \ldots, k_{n+1}\right) \mapsto m\left(k_{0}, \ldots, k_{n+1}\right)$ has a support as in Point i) of Definition 3.1 and is bounded by the right member of (24).

The parameter $\tau$ which appears in the exponent of $k_{0}$ plays an important role because of the following boundedness estimates.

Proposition 3.2. - Consider $\nu, \delta, \tau, s>0$ there is $s_{0}=s_{0}(\alpha, \nu)>0$ such that any $\mathcal{M} \in \mathcal{M}_{\alpha, n, \delta}^{\tau, \nu}$ admits an unique extension as a bounded operator from $\left(\mathcal{H}_{\alpha}^{s}\right)^{n}$ to $\mathcal{L}\left(\mathcal{H}_{\alpha}^{s}, \mathcal{H}_{\alpha}^{s-\tau-\frac{1}{\alpha}}\right)$ for $s>s_{0}$. In other words, one has for all $u_{1}, \ldots, u_{n+1} \in \mathcal{S}(\mathbb{R})$ the following inequality

$$
\left\|\mathcal{M}\left(u_{1}, \ldots, u_{n}\right) u_{n+1}\right\|_{\mathcal{H}_{\alpha}^{s-\tau-\frac{1}{\alpha}}} \leq C \prod_{j=1}^{n+1}\left\|u_{j}\right\|_{\mathcal{H}_{\alpha}^{s}}
$$

REMARK 3.3. - If $\tau \leq 2 s-\frac{1}{\alpha}$ holds then any element $\mathcal{M} \in \mathcal{M}_{\alpha, n, \delta}^{\tau, \nu}$ satisfies the $\mathcal{H}_{\alpha}^{s}$ boundedness property

$$
\left|\left\langle\mathcal{M}\left(u_{1}, \ldots, u_{n}\right) u_{n+1}, u_{0}\right\rangle\right| \leq C \prod_{j=0}^{n+1}\left\|u_{j}\right\|_{H_{\alpha}^{s}} .
$$

The proof of Proposition 3.2 needs the following lemma

LEMmA 3.4. - For all integer $k_{0} \geq 1$ and real numbers $A \geq 1, \omega>\frac{1}{\alpha^{\prime}}$ one has

$$
\sum_{k_{n+1} \geq 1} \frac{1}{\left(\left|k_{n+1}^{\alpha^{\prime}}-k_{0}^{\alpha^{\prime}}\right|+A^{\alpha^{\prime}}\right)^{\omega}} \leq C k_{0}^{\frac{1}{\alpha+1}} A^{1-\alpha^{\prime} \omega} .
$$

Proof. - We consider three cases. If $1 \leq k_{n+1} \leq k_{0}$ then

$$
\left|k_{n+1}^{\alpha^{\prime}}-k_{0}^{\alpha^{\prime}}\right| \geq \alpha^{\prime} \frac{k_{0}-k_{n+1}}{k_{0}^{1-\alpha^{\prime}}}=\alpha^{\prime} \frac{k_{0}-k_{n+1}}{k_{0}^{\frac{1}{\alpha+1}}} .
$$

Using (13), one has

$$
\begin{aligned}
\sum_{k_{n+1}=1}^{k_{0}} \frac{1}{\left(\left|k_{n+1}^{\alpha^{\prime}}-k_{0}^{\alpha^{\prime}}\right|+A^{\alpha^{\prime}}\right)^{\omega}} & \leq \sum_{k_{n+1}=1}^{k_{0}} \frac{k_{0}^{\omega\left(1-\alpha^{\prime}\right)}}{\left(\left|k_{0}-k_{n+1}\right|+k_{0}^{\frac{1}{\alpha+1}} A^{\alpha^{\prime}}\right)^{\omega}} \\
& \leq k_{0}^{\omega\left(1-\alpha^{\prime}\right)} \sum_{v \geq 0} \frac{1}{\left(v+k_{0}^{\frac{1}{\alpha+1}} A^{\alpha^{\prime}}\right)^{\omega}} \\
& \leq \frac{k_{0}^{\omega\left(1-\alpha^{\prime}\right)}}{\left(k_{0}^{\frac{1}{\alpha+1}} A^{\alpha^{\prime}}\right)^{\omega-1}} \leq k_{0}^{\frac{1}{\alpha+1}} A^{1-\alpha^{\prime} \omega}
\end{aligned}
$$


If $k_{0} \leq k_{n+1} \leq 2 k_{0}$ then the following inequality

$$
\sup _{0<x<1} \frac{\left|1-(1-x)^{\alpha^{\prime}}\right|}{(1+x)^{\alpha^{\prime}}-1}<+\infty
$$

which leads to

$$
\forall \ell \in\left[0, k_{0}\right] \quad\left|k_{0}^{\alpha^{\prime}}-\left(k_{0}-\ell\right)^{\alpha^{\prime}}\right| \leq C\left|\left(k_{0}+\ell\right)^{\alpha^{\prime}}-k_{0}^{\alpha^{\prime}}\right|
$$

and so

$$
\sum_{k_{n+1}=k_{0}}^{2 k_{0}} \frac{1}{\left(\left|k_{0}^{\alpha^{\prime}}-k_{n+1}^{\alpha^{\prime}}\right|+A^{\alpha^{\prime}}\right)^{\omega}} \leq C \sum_{k_{n+1}=1}^{k_{0}} \frac{1}{\left(\left|k_{0}^{\alpha^{\prime}}-k_{n+1}^{\alpha^{\prime}}\right|+A^{\alpha^{\prime}}\right)^{\omega}} .
$$

We are back to the first case. If $k_{n+1} \geq 2 k_{0}$, then we have

$$
\begin{aligned}
\sum_{k_{n+1} \geq 2 k_{0}} \frac{1}{\left(\left|k_{0}^{\alpha^{\prime}}-k_{n+1}^{\alpha^{\prime}}\right|+A^{\alpha^{\prime}}\right) \omega} & \leq \sum_{k_{n+1} \geq 2 k_{0}} \frac{1}{\left(k_{n+1}^{\alpha^{\prime}}+A^{\alpha^{\prime}}\right)^{\omega}} \\
& \leq \sum_{k_{n+1} \geq 1} \frac{1}{\left(k_{n+1}+A\right)^{\alpha^{\prime} \omega}} \\
& \leq \frac{1}{A^{\alpha^{\prime} \omega-1}} \\
& \leq k_{0}^{\frac{1}{\alpha+1}} A^{1-\alpha^{\prime} \omega} .
\end{aligned}
$$

We can now prove Proposition 3.2.

Proof. - Define

$\Omega(\delta)=\left\{\left(k_{1}, \ldots, k_{n+1}\right) \in\left(\mathbb{N}^{\star}\right)^{n+1}, \quad \delta \leq \frac{k_{n+1}}{k_{0}} \leq \frac{1}{\delta}, \quad k_{n+1} \geq \max \left(k_{1}, \ldots, k_{n}\right)\right\}$,

$\Upsilon(\delta)=\left\{\left(k_{1}, \ldots, k_{n+1}\right) \in \Omega(\delta), \quad k_{1} \leq \cdots \leq k_{n+1}\right\}$.

We want to prove

$$
\left\|\mathcal{M}\left(u_{1}, \ldots, u_{n}\right) u_{n+1}\right\|_{\mathcal{H}_{\alpha}^{s-\tau-\frac{1}{\alpha}}}^{2} \leq C \prod_{j=1}^{n+1}\left\|u_{j}\right\|_{H_{\alpha}^{s}}^{2} .
$$

By definition, the left side is

$$
\sum_{k_{0} \geq 1} k_{0}^{2 \alpha^{\prime}\left(s-\tau-\frac{1}{\alpha}\right)}\left|\sum_{k_{1}, \ldots, k_{n+1}}\left\langle M\left(\phi_{\alpha, k_{1}}, \ldots, \phi_{\alpha, k_{n}}\right) \phi_{\alpha, k_{n+1}}, \phi_{\alpha, k_{0}}\right\rangle \prod_{j=1}^{n+1}\left\langle u_{j}, \phi_{\alpha, k_{j}}\right\rangle\right|^{2} .
$$

Using the definition (3.1), the previous term is dominated by

$$
\sum_{k_{0} \geq 1} k_{0}^{2 \alpha^{\prime}\left(s-\tau-\frac{1}{\alpha}\right)}\left[\sum_{\Omega(\delta)} C_{N} \max \left(k_{1}^{\alpha^{\prime} \nu}, \ldots, k_{n}^{\alpha^{\prime} \nu}\right) k_{0}^{\alpha^{\prime} \tau}\right.
$$




$$
\left.\left(\frac{\max \left(k_{1}^{\alpha^{\prime}}, \ldots, k_{n}^{\alpha^{\prime}}\right)}{\max \left(k_{1}, \ldots, k_{n}^{\alpha^{\prime}}+\left|k_{0}^{\alpha^{\prime}}-k_{n+1}^{\alpha^{\prime}}\right|\right.}\right)^{N} \prod_{j=1}^{n+1}\left|\left\langle u_{j}, \phi_{\alpha, k_{j}}\right\rangle\right|\right]^{2} .
$$

In the sequel, we will only use the condition $N>\frac{1}{\alpha^{\prime}}$ which will be necessary to apply Lemma 3.4. By symmetry of the variables $k_{1}, \ldots, k_{n+1}$, one can replace $\Omega(\delta)$ by $\Upsilon(\delta)$. Thus, it suffices to consider

$$
\begin{aligned}
& \sum_{k_{0} \geq 1} k_{0}^{2 \alpha^{\prime}\left(s-\tau-\frac{1}{\alpha}\right)}\left[\sum_{\Upsilon(\delta)} C_{N} k_{n}^{\alpha^{\prime} \nu} k_{0}^{\alpha^{\prime} \tau}\left(\frac{k_{n}^{\alpha^{\prime}}}{k_{n}^{\alpha^{\prime}}+\left|k_{0}^{\alpha^{\prime}}-k_{n+1}^{\alpha^{\prime}}\right|}\right)^{N} \prod_{j=1}^{n+1}\left|\left\langle u_{j}, \phi_{\alpha, k_{j}}\right\rangle\right|\right]^{2} \\
= & C_{N} \sum_{k_{0} \geq 1} k_{0}^{2 \alpha^{\prime}\left(s-\frac{1}{\alpha}\right)}\left[\sum_{\Upsilon(\delta)} k_{n}^{\alpha^{\prime} \nu}\left(\frac{k_{n}^{\alpha^{\prime}}}{k_{n}^{\alpha^{\prime}}+\left|k_{0}^{\alpha^{\prime}}-k_{n+1}^{\alpha^{\prime}}\right|}\right)^{N} \prod_{j=1}^{n+1}\left|\left\langle u_{j}, \phi_{\alpha, k_{j}}\right\rangle\right|\right]^{2} .
\end{aligned}
$$

The Cauchy-Schwarz inequality allows us to bound the previous term by

$$
\begin{aligned}
& \leq C_{N} \sum_{k_{0} \geq 1} k_{0}^{2 \alpha^{\prime}\left(s-\frac{1}{\alpha}\right)}\left[\sum_{\Upsilon(\delta)} k_{n}^{\alpha^{\prime} \nu}\left(\frac{k_{n}^{\alpha^{\prime}}}{k_{n}^{\alpha^{\prime}}+\left|k_{0}^{\alpha^{\prime}}-k_{n+1}^{\alpha^{\prime}}\right|}\right)^{N} \prod_{j=1}^{n}\left|\left\langle u_{j}, \phi_{\alpha, k_{j}}\right\rangle\right|\right] \\
& {\left[\sum_{\Upsilon(\delta)} k_{n}^{\alpha^{\prime} \nu}\left(\frac{k_{n}^{\alpha^{\prime}}}{k_{n}^{\alpha^{\prime}}+\left|k_{0}^{\alpha^{\prime}}-k_{n+1}^{\alpha^{\prime}}\right|}\right)^{N}\left|\left\langle u_{n+1}, \phi_{\alpha, k_{n+1}}\right\rangle\right|^{2} \prod_{j=1}^{n}\left|\left\langle u_{j}, \phi_{\alpha, k_{j}}\right\rangle\right|\right] .}
\end{aligned}
$$

With the help of Lemma 3.4, one gets

$$
\begin{aligned}
& \sum_{\Upsilon(\delta)} k_{n}^{\alpha^{\prime} \nu}\left(\frac{k_{n}^{\alpha^{\prime}}}{k_{n}^{\alpha^{\prime}}+\left|k_{0}^{\alpha^{\prime}}-k_{n+1}^{\alpha^{\prime}}\right|}\right)^{N} \prod_{j=1}^{n}\left|\left\langle u_{j}, \phi_{\alpha, k_{j}}\right\rangle\right| \\
\leq & \sum_{k_{1}, \ldots, k_{n}} k_{n}^{\alpha^{\prime} \nu} k_{0}^{\frac{1}{\alpha+1}} k_{n} \prod_{j=1}^{n}\left|\left\langle u_{j}, \phi_{\alpha, k_{j}}\right\rangle\right| \\
\leq & k_{0}^{\frac{1}{\alpha+1}} \prod_{j=1}^{n}\left(\sum_{k_{j} \geq 1} k_{j}^{1+\alpha^{\prime} \nu} \mid\left\langle u_{j}, \phi_{\alpha, k_{j}}\right\rangle\right) \\
\leq & k_{0}^{\frac{1}{\alpha+1}} \prod_{j=1}^{n}\left\|u_{j}\right\|_{\mathcal{H}_{\alpha}^{s}},
\end{aligned}
$$

provided that $s \geq \frac{2+\alpha^{\prime} \nu}{\alpha^{\prime}}$ holds. Consequently, $\left\|\mathcal{M}\left(u_{1}, \ldots, u_{n}\right) u_{n+1}\right\|_{\mathcal{H}_{\alpha}^{s-\tau-\frac{1}{\alpha}}}^{2}$ is bounded by

$$
\sum_{\substack{k_{0} \geq 1 \\ \Upsilon(\delta)}} \frac{k_{0}^{2 \alpha^{\prime}\left(s-\frac{1}{\alpha}\right)+\frac{1}{\alpha+1}} k_{n}^{\alpha^{\prime}(\nu+N)}}{\left(k_{n}^{\alpha^{\prime}}+\left|k_{0}^{\alpha^{\prime}}-k_{n+1}^{\alpha^{\prime}}\right|\right)^{N}}\left|\left\langle u_{n+1}, \phi_{\alpha, k_{n+1}}\right\rangle\right|^{2} \prod_{j=1}^{n}\left|\left\langle u_{j}, \phi_{\alpha, k_{j}}\right\rangle\right| \times \|\left. u_{j}\right|_{\mathcal{H}_{\alpha}^{s}} .
$$


Remember now that in the set $\Upsilon(\delta)$ one has $k_{n+1} \simeq k_{0}$ (see (25)). Hence, we get the bound

$$
\leq\left(\prod_{j=1}^{n}\left\|u_{j}\right\|_{\mathcal{H}_{\alpha}^{s}}\right) \sum_{\substack{k_{0} \geq 1 \\ \Upsilon(\delta)}} \frac{k_{n+1}^{2 \alpha^{\prime}\left(s-\frac{1}{\alpha}\right)+\frac{1}{\alpha+1}} k_{n}^{\alpha^{\prime}(\nu+N)}}{\left(k_{n}^{\alpha^{\prime}}+\left|k_{0}^{\alpha^{\prime}}-k_{n+1}^{\alpha^{\prime}}\right|\right)^{N}}\left|\left\langle u_{n+1}, \phi_{\alpha, k_{n+1}}\right\rangle\right|^{2} \prod_{j=1}^{n}\left|\left\langle u_{j}, \phi_{\alpha, k_{j}}\right\rangle\right| .
$$

Using a new time Lemma 3.4 (and inverting $k_{0}$ and $k_{n+1}$ ), we can bound :

$$
\begin{aligned}
\leq & C\left(\prod_{j=1}^{n}\left\|u_{j}\right\|_{\mathcal{H}_{\alpha}^{s}}\right) \sum_{\Upsilon(\delta)}\left[k_{n+1}^{\frac{1}{\alpha+1}} k_{n}^{1-\alpha^{\prime} N} k_{n+1}^{2 \alpha^{\prime}\left(s-\frac{1}{\alpha}\right)+\frac{1}{\alpha+1}} k_{n}^{\alpha^{\prime}(\nu+N)}\right. \\
& \left.\left|\left\langle u_{n+1}, \phi_{\alpha, k_{n+1}}\right\rangle\right|^{2} \prod_{j=1}^{n}\left|\left\langle u_{j}, \phi_{\alpha, k_{j}}\right\rangle\right|\right] \\
= & \left(\prod_{j=1}^{n}\left\|u_{j}\right\|_{\mathcal{H}_{\alpha}^{s}}\right) \sum_{\Upsilon(\delta)} k_{n+1}^{2 \alpha^{\prime}\left(s-\frac{1}{\alpha}\right)+\frac{2}{\alpha+1}} k_{n}^{1+\alpha^{\prime} \nu}\left|\left\langle u_{n+1}, \phi_{\alpha, k_{n+1}}\right\rangle\right|^{2} \prod_{j=1}^{n}\left|\left\langle u_{j}, \phi_{\alpha, k_{j}}\right\rangle\right| .
\end{aligned}
$$

By definition, $\alpha^{\prime}$ equals $\frac{\alpha}{\alpha+1}$. Thus, we have

$$
\begin{aligned}
& \left(\prod_{j=1}^{n}\left\|u_{j}\right\|_{\mathcal{H}_{\alpha}^{s}}\right) \sum_{\Upsilon(\delta)} k_{n+1}^{2 \alpha^{\prime} s}\left|\left\langle u_{n+1}, \phi_{\alpha, k_{n+1}}\right\rangle\right|^{2} \prod_{j=1}^{n} k_{j}^{1+\alpha^{\prime} \nu}\left|\left\langle u_{j}, \phi_{\alpha, k_{j}}\right\rangle\right| \\
& \leq\left(\prod_{j=1}^{n}\left\|u_{j}\right\|_{\mathcal{H}_{\alpha}^{s}}\right)\left\|u_{n+1}\right\|_{\mathcal{H}_{\alpha}^{s}}^{2} \sum_{k_{1}, \ldots, k_{n}} k_{j}^{1+\alpha^{\prime} \nu}\left|\left\langle u_{j}, \phi_{\alpha, k_{j}}\right\rangle\right| .
\end{aligned}
$$

To conclude, notice that if $s$ is enough large, say $s \geq \frac{2+\alpha^{\prime} \nu}{\alpha^{\prime}}$, then

$$
\begin{aligned}
& \sum_{k_{1}, \ldots, k_{n}} k_{j}^{1+\alpha^{\prime} \nu}\left|\left\langle u_{j}, \phi_{\alpha, k_{j}}\right\rangle\right| \leq \sum_{k_{1}, \ldots, k_{n}} \frac{1}{k_{j}} k_{j}^{2+\alpha^{\prime} \nu}\left|\left\langle u_{j}, \phi_{\alpha, k_{j}}\right\rangle\right| \\
& \leq C \prod_{j=1}^{n}\left(\sum_{k_{j} \geq 1} k_{j}^{2\left(2+\alpha^{\prime} \nu\right)}\left|\left\langle u_{j}, \phi_{\alpha, k_{j}}\right\rangle\right|^{2}\right)^{\frac{1}{2}} \leq C \prod_{j=1}^{n}\left\|u_{j}\right\|_{\mathcal{H}_{\alpha}^{s}} .
\end{aligned}
$$

Combining our estimates, we have proved

$$
\left\|\mathcal{M}\left(u_{1}, \ldots, u_{n}\right) u_{n+1}\right\|_{\mathcal{H}_{\alpha}^{s-\tau-\frac{1}{\alpha}}} \leq C \prod_{j=1}^{n+1}\left\|u_{j}\right\|_{\mathcal{H}_{\alpha}^{s}} .
$$

In the procedure of normal form, some term will be irrelevant to eliminate. That is why we define nonresonant multilinear operators. 
Definition 3.5. - Let $\mathcal{M}$ be an operator in $\mathcal{M}_{\alpha, n, \delta}^{\tau, \nu}$ and $\ell$ be an integer in $\in[0, n+1]$. We write $\mathcal{M} \in \mathcal{M}_{\alpha, n, \delta}^{\tau, \nu}[\ell]$ if for all $\left(k_{0}, \ldots, k_{n+1}\right) \in(\mathbb{N} \backslash\{0\})^{n+2}$

$$
\left\{k_{0}, \ldots, k_{\ell}\right\}=\left\{k_{\ell+1}, \ldots, k_{n+1}\right\} \Rightarrow\left\langle\mathcal{M}\left(\phi_{\alpha, k_{1}}, \ldots, \phi_{\alpha, k_{n}}\right) \phi_{\alpha, k_{n+1}}, \phi_{\alpha, k_{0}}\right\rangle=0 .
$$

We also write $\mathcal{M} \in \mathcal{M}_{\alpha, n, \delta}^{\tau, \nu}[\widehat{\ell}]$ if for all $\left(k_{0}, \ldots, k_{n+1}\right) \in(\mathbb{N} \backslash\{0\})^{n+2}$ $\left\{k_{n+1}, k_{0}, \ldots, k_{\ell}\right\}=\left\{k_{\ell+1}, \ldots, k_{n}\right\} \Rightarrow\left\langle\mathcal{M}\left(\phi_{\alpha, k_{1}}, \ldots, \phi_{\alpha, k_{n}}\right) \phi_{\alpha, k_{n+1}}, \phi_{\alpha, k_{0}}\right\rangle=0$.

3.2. A second class of multilinear operators. - Let us define other multilinear operators.

Definition 3.6. - A multilinear operator $\mathcal{R}: \mathcal{S}(\mathbb{R})^{n} \rightarrow \mathcal{L}\left(\mathcal{S}(\mathbb{R}), \mathcal{S}^{\prime}(\mathbb{R})\right)$ is in the class $\mathcal{R}_{\alpha, n}^{\tau, \nu}$ if the following estimates hold for all $\left(k_{0}, \ldots, k_{n+1}\right) \in$ $(\mathbb{N} \backslash\{0\})^{n+2}$ and $N>0$

$$
\left|\left\langle\mathcal{R}\left(\phi_{\alpha, k_{1}}, \ldots, \phi_{\alpha, k_{n}}\right) \phi_{\alpha, k_{n+1}}, \phi_{\alpha, k_{0}}\right\rangle\right| \leq C_{N} k_{0}^{\alpha^{\prime} \tau} \frac{\max _{2}\left(k_{1}, \ldots, k_{n+1}\right)^{\nu+N}}{\left(k_{0}+\cdots+k_{n+1}\right)^{N}} .
$$

As for the space $\mathcal{M}_{\alpha, n, \delta}^{\tau, \nu}$, we have a boundedness property for spaces $\mathcal{R}_{\alpha, n}^{\tau, \nu}$.

Proposition 3.7. - Consider $\nu, \tau, s>0$, there is $s_{0}=s_{0}(\alpha, \nu)>0$ such that if $s$ and $3 s-\tau$ are larger than $s_{0}(\alpha, \nu)$ then any operator $\mathcal{R} \in \mathcal{R}_{\alpha, n}^{\tau, \nu}$ admits an unique extension as a bounded operator from $\left(\mathcal{H}_{\alpha}^{s}\right)^{n}$ to $\mathcal{L}\left(\mathcal{H}_{\alpha}^{s}, \mathcal{H}_{\alpha}^{-s}\right)$. In other words, the following inequality holds for all $u_{1}, \ldots, u_{n+1} \in \mathcal{S}(\mathbb{R})$

$$
\left\|\mathcal{R}\left(u_{1}, \ldots, u_{n}\right) u_{n+1}\right\|_{\mathcal{H}_{\alpha}^{-s}} \leq C \prod_{j=1}^{n+1}\left\|u_{j}\right\|_{\mathcal{H}_{\alpha}^{s}} .
$$

Proof. - Let us prove that there is $s_{0}(\alpha, \nu)$ such that if $s>s_{0}$ then

$$
\left\|\mathcal{R}\left(u_{1}, \ldots, u_{n}\right) u_{n+1}\right\|_{\mathcal{H}_{\alpha}^{2 s-\nu-\tau-\frac{4}{\alpha^{\prime}}}} \leq C \prod_{j=1}^{n+1}\left\|u_{j}\right\|_{\mathcal{H}_{\alpha}^{s}} .
$$

It is easy to see that (26) will be a consequence. Indeed, we can suppose $s_{0}(\alpha, \nu)$ to be larger than $\nu+\frac{4}{\alpha^{\prime}}$ in $(27)$. Thus if $3 s-\tau \geq s_{0}(\alpha, \nu)$ holds then $2 s-\nu-\tau-\frac{4}{\alpha^{\prime}} \geq-s$ also holds and one sees that (27) implies (26). The square of the left member of (27) is

$$
\sum_{k_{0} \geq 1} k_{0}^{2 \alpha^{\prime}\left(2 s-\tau-\nu-4 / \alpha^{\prime}\right)}\left|\sum\left\langle\mathcal{R}\left(\phi_{\alpha, k_{1}}, \ldots, \phi_{\alpha, k_{n}}\right) \phi_{\alpha, k_{n+1}}, \phi_{\alpha, k_{0}}\right\rangle \prod_{j=1}^{n+1}\left\langle\phi_{\alpha, k_{j}}, u_{j}\right\rangle\right|^{2} .
$$

Let us choose some parameters : the regularity $s$ has to satisfy

$$
s>s_{0}:=\max \left(\frac{1}{\alpha^{\prime}}, \frac{\nu+\frac{3}{\alpha^{\prime}}}{2}, \nu+\frac{4}{\alpha^{\prime}}\right) .
$$


Then define $N=2 s-\nu-\frac{2}{\alpha^{\prime}}$. One has obviously $N>\frac{1}{\alpha^{\prime}}$. Those choices are not sharp for our proof but are sufficient. The definition of spaces $\mathcal{R}_{\alpha, n}^{\tau, \nu}$ implies that (28) is bounded by

$$
\begin{aligned}
& C \sum_{k_{0} \geq 1} k_{0}^{2 \alpha^{\prime}\left(2 s-\nu-4 / \alpha^{\prime}\right)}\left|\sum_{k_{1} \leq \cdots \leq k_{n+1}} \frac{k_{n}^{\alpha^{\prime}(\nu+N)}}{\left(k_{0}^{\alpha^{\prime}}+\cdots+k_{n+1}^{\alpha^{\prime}}\right)^{N}} \prod_{j=1}^{n+1}\right|\left\langle\phi_{\alpha, k_{j}}, u_{j}\right\rangle||^{2} \\
\leq & C \sum_{k_{0} \geq 1} k_{0}^{2 \alpha^{\prime}\left(2 s-\nu-4 / \alpha^{\prime}\right)}\left|\sum_{k_{1} \leq \cdots \leq k_{n+1}} \frac{k_{n}^{\alpha^{\prime}(\nu+N)}}{\left(k_{0}^{\alpha^{\prime}}+k_{n+1}^{\alpha^{\prime}}\right)^{N}} \prod_{j=1}^{n+1} \frac{\left\|u_{j}\right\|_{\mathcal{H}_{\alpha}^{s}}}{k_{j}^{\alpha^{\prime} s}}\right|^{2} \\
\leq & C\left[\prod_{j=1}^{n+1}\left\|u_{j}\right\|_{\mathcal{H}_{\alpha}^{s}}^{2}\right] \sum_{k_{0} \geq 1} k_{0}^{2 \alpha^{\prime}\left(2 s-\nu-4 / \alpha^{\prime}\right)}\left|\sum_{k_{1} \leq \cdots \leq k_{n+1}} \frac{k_{n}^{\alpha^{\prime}(\nu+N-2 s)}}{\left(k_{0}^{\alpha^{\prime}}+k_{n+1}^{\alpha^{\prime}}\right)^{N}} \prod_{j=1}^{n-1} \frac{1}{k_{j}^{\alpha^{\prime} s}}\right|^{2} .
\end{aligned}
$$

As $N>\frac{1}{\alpha^{\prime}}$, one can use Lemma 3.4 (with $k_{0}=1$ ) to bound the previous term by :

$$
\begin{aligned}
& C\left[\prod_{j=1}^{n+1}\left\|u_{j}\right\|_{\mathcal{H}_{\alpha}^{s}}^{2}\right] \sum_{k_{0} \geq 1} k_{0}^{2 \alpha^{\prime}\left(2 s-\nu-4 / \alpha^{\prime}\right)}\left|\sum_{k_{1} \leq \cdots \leq k_{n}} \frac{k_{n}^{\alpha^{\prime}(\nu+N-2 s)}}{k_{0}^{\alpha^{\prime} N-1}} \prod_{j=1}^{n-1} \frac{1}{k_{j}^{\alpha^{\prime} s}}\right|^{2} \\
\leq & C\left[\prod_{j=1}^{n+1}\left\|u_{j}\right\|_{\mathcal{H}_{\alpha}^{s}}^{2}\right]\left[\sum_{k_{0} \geq 1} \frac{k_{0}^{2 \alpha^{\prime}\left(2 s-\nu-4 / \alpha^{\prime}\right)}}{k_{0}^{2 \alpha^{\prime} N-2}}\right]\left[\sum_{k \geq 1} \frac{1}{k^{\alpha^{\prime} s}}\right]^{n-1} \sum_{k_{n} \geq 1} \frac{1}{k_{n}^{\alpha^{\prime}(2 s-N-\nu)}} .
\end{aligned}
$$

Every sum is convergent since

i) $s>\frac{1}{\alpha^{\prime}}$,

ii) $2 s-N-\nu=\frac{2}{\alpha^{\prime}}>\frac{1}{\alpha^{\prime}}$,

iii) $2 \alpha^{\prime} N-2-2 \alpha^{\prime}\left(2 s-\nu-\frac{4}{\alpha^{\prime}}\right)=-4-2+8=2>1$.

We also define nonresonant operators in the space $\mathcal{R}_{\alpha, n}^{\tau, \nu}$.

Definition 3.8. - Let $\mathcal{R}$ be an operator in $\mathcal{R}_{\alpha, n}^{\tau, \nu}$ and $\ell$ be an integer in $\in[0, n+1]$. We write $\mathcal{R} \in \mathcal{R}_{\alpha, n}^{\tau, \nu}[\ell]$ if for all $\left(k_{0}, \ldots, k_{n+1}\right) \in(\mathbb{N} \backslash\{0\})^{n+2}$

$$
\left\{k_{0}, \ldots, k_{\ell}\right\}=\left\{k_{\ell+1}, \ldots, k_{n+1}\right\} \Rightarrow\left\langle\mathcal{R}\left(\phi_{\alpha, k_{1}}, \ldots, \phi_{\alpha, k_{n}}\right) \phi_{\alpha, k_{n+1}}, \phi_{\alpha, k_{0}}\right\rangle=0 .
$$

We also write $\mathcal{R} \in \mathcal{R}_{\alpha, n}^{\tau, \nu}[\widehat{\ell \ell}]$ if for all $\left(k_{0}, \ldots, k_{n+1}\right) \in(\mathbb{N} \backslash\{0\})^{n+2}$

$$
\left\{k_{n+1}, k_{0}, \ldots, k_{\ell}\right\}=\left\{k_{\ell+1}, \ldots, k_{n}\right\} \Rightarrow\left\langle\mathcal{R}\left(\phi_{\alpha, k_{1}}, \ldots, \phi_{\alpha, k_{n}}\right) \phi_{\alpha, k_{n+1}}, \phi_{\alpha, k_{0}}\right\rangle=0 .
$$

\section{Decomposing the nonlinearity}

The main objective of the next subsections is to prove the following result which involves multilinear models. 
Proposition 4.1. - For each integer $\ell \in[0, n]$, there are

$$
\mathcal{M}_{\ell} \in \mathcal{M}_{\alpha, n, \delta}^{2 s-2, \nu}[\ell], \quad \widehat{\mathcal{M}}_{\ell} \in \mathcal{M}_{\alpha, n, \delta}^{2 s-1, \nu}[\widehat{\ell}], \quad \mathcal{R}_{\ell} \in \mathcal{R}_{\alpha, n}^{2 s, \nu}[\ell], \quad \widehat{\mathcal{R}}_{\ell} \in \mathcal{R}_{\alpha, n}^{2 s, \nu}[\widehat{\ell}]
$$

such that

$$
\begin{aligned}
\frac{d}{d t} \Theta_{s}(u(t))= & \sum_{\ell=0}^{n} \operatorname{Re} i\left\langle\mathcal{M}_{\ell}(\underbrace{\bar{u}, \ldots, \bar{u}}_{\ell}, \underbrace{u, \ldots, u}_{n-\ell}) u\right\rangle \\
& +\sum_{\ell=0}^{n} \operatorname{Re} i\left\langle\mathcal{R}_{\ell}(\underbrace{\bar{u}, \ldots, \bar{u}}_{\ell}, \underbrace{u, \ldots, u}_{n-\ell}) u, u\right\rangle \\
& +\sum_{\ell=0}^{n} \operatorname{Re} i\left\langle\widehat{M}_{\ell}(\underbrace{\bar{u}, \ldots, \bar{u}}_{\ell}, \underbrace{u, \ldots, u}_{n-\ell}) \bar{u}, u\right\rangle \\
& +\sum_{\ell=0}^{n} \operatorname{Re} i\left\langle\widehat{\mathcal{R}}_{\ell}(\underbrace{\bar{u}, \ldots, \bar{u}}_{\ell}, \underbrace{u, \ldots, u}_{n-\ell}) \bar{u}, u\right\rangle .
\end{aligned}
$$

4.1. Introduction of two terms. - We first decompose $w$ on the eigenbasis $\left(\phi_{\alpha, k}\right)_{k \geq 1}$ of $L^{2}(\mathbb{R})$ :

$$
\begin{aligned}
w^{n+1} & =\left(\sum_{k \geq 1}\left\langle w, \phi_{\alpha, k}\right\rangle \phi_{\alpha, k}\right)^{n+1} \\
& =\sum_{k_{1}, \ldots, k_{n+1} \geq 1}\left\langle w, \phi_{\alpha, k_{1}}\right\rangle \cdots\left\langle w, \phi_{\alpha, k_{n+1}}\right\rangle \prod_{j=1}^{n+1} \phi_{\alpha, k_{j}} .
\end{aligned}
$$

By symmetry, there is a map $b:(\mathbb{N} \backslash\{0\})^{n+1} \rightarrow \mathbb{Q}$ which is symmetric with respect to the first $n$ variables, has support in

$$
\Gamma:=\left\{\left(k_{1}, \ldots, k_{n+1}\right), \quad \max \left(k_{1}, \ldots, k_{n}\right) \leq k_{n+1}\right\}
$$

and satisfies

$$
w^{n+1}=\sum_{\Gamma} b\left(k_{1}, \ldots, k_{n}, k_{n+1}\right) \prod_{j=1}^{n+1}\left\langle w, \phi_{\alpha, k_{j}}\right\rangle \phi_{\alpha, k_{j}}
$$

Indeed if $\overline{\left(k_{1}, \ldots, k_{n+1}\right)}$ is the ordered uplet of $\left(k_{1}, \ldots, k_{n+1}\right)$, then one can check that $b\left(k_{1}, \ldots, k_{n+1}\right)$ is nothing else than

$$
\frac{\operatorname{Card}\left\{\left(\ell_{1}, \ldots, \ell_{n+1}\right) \in(\mathbb{N} \backslash\{0\})^{n+1}, \quad \overline{\left(\ell_{1}, \ldots, \ell_{n+1}\right)}=\overline{\left(k_{1}, \ldots, k_{n+1}\right)}\right\}}{\operatorname{Card}\left\{\left(\ell_{1}, \ldots, \ell_{n}\right) \in(\mathbb{N} \backslash\{0\})^{n}, \quad \overline{\left(\ell_{1}, \ldots, \ell_{n}, k_{n+1}\right)}=\overline{\left(k_{1}, \ldots, k_{n+1}\right)}\right\}} .
$$

For instance, if $\max \left(k_{1}, \ldots, k_{n}\right)<k_{n+1}$ then $b\left(k_{1}, \ldots, k_{n+1}\right)=n+1$. Remember now that $w$ and $u$ are related by the equality

$$
w=\Lambda_{\alpha, m}^{-1}\left(\frac{u+\bar{u}}{2}\right)=\frac{1}{2} \Lambda_{\alpha, m}^{-1} u+\frac{1}{2} \Lambda_{\alpha, m}^{-1} \bar{u} .
$$


Thus, it is possible to write $(2 w)^{n+1}$ as

$$
\sum_{\Gamma} \sum_{F \subset[1, n+1]} b\left(k_{1}, \ldots, k_{n+1}\right) \prod_{j \in F}\left\langle\Lambda_{\alpha, m}^{-1} \bar{u}, \phi_{\alpha, k_{j}}\right\rangle \prod_{j \notin F}\left\langle\Lambda_{\alpha, m}^{-1} u, \phi_{\alpha, k_{j}}\right\rangle\left[\prod_{j=1}^{n+1} \phi_{\alpha, k_{j}}\right] \text {. }
$$

Separating the subsets $F$ which contain $n+1$, we can compute as follows

$$
w^{n+1}=-\sum_{\ell=0}^{n} C_{\ell}(\underbrace{\bar{u}, \ldots, \bar{u}}_{\ell}, \underbrace{u, \ldots, u}_{n-\ell}) u-\sum_{\ell=0}^{n} C_{\ell}(\underbrace{\bar{u}, \ldots, \bar{u}}_{\ell}, \underbrace{u, \ldots, u}_{n-\ell}) \bar{u} .
$$

where the symmetry of $b$ of the variables $k_{1}, \ldots, k_{n}$ gives the following definition

$$
\begin{gathered}
C_{\ell}(\underbrace{\bar{u}, \ldots, \bar{u}}_{\ell}, \underbrace{u, \ldots, u}_{n-\ell}) f:=\frac{1}{2^{n+1}}\left(\begin{array}{c}
n \\
\ell
\end{array}\right) \sum_{\Gamma}\left\{b\left(k_{1}, \ldots, k_{n+1}\right)\left[\prod_{j=1}^{n+1} \phi_{\alpha, k_{j}}\right]\right. \\
\left.\left[\prod_{j=1}^{\ell}\left\langle\Lambda_{\alpha, m}^{-1} \bar{u}, \phi_{\alpha, k_{j}}\right\rangle\right]\left[\prod_{j=\ell+1}^{n}\left\langle\Lambda_{\alpha, m}^{-1} u, \phi_{\alpha, k_{j}}\right\rangle\right]\left\langle\Lambda_{\alpha, m}^{-1} f, \phi_{\alpha, k_{n+1}}\right\rangle\right\} .
\end{gathered}
$$

Let us plug (29) in (6) :

$$
\begin{aligned}
& \frac{d}{d t} \Theta_{s}(u(t))=\sum_{\ell=0}^{n} \operatorname{Re} i\left\langle\Lambda_{\alpha, m}^{s} C_{\ell}(\underbrace{\bar{u}, \ldots, \bar{u}}_{\ell}, \underbrace{u, \ldots, u}_{n-\ell}) u, \Lambda_{\alpha, m}^{s} u\right\rangle \\
& +\sum_{\ell=0}^{n} \operatorname{Re} i\left\langle\Lambda_{\alpha, m}^{s} C_{\ell}(\underbrace{\bar{u}, \ldots, \bar{u}}_{\ell}, \underbrace{u, \ldots, u}_{n-\ell}) \bar{u}, \Lambda_{\alpha, m}^{s} u\right\rangle .
\end{aligned}
$$

Proposition 4.1 will be a direct consequence of the following two lemmas :

LemMA 4.2. - For each integer $\ell \in[0, n]$, there are $\mathcal{M}_{\ell} \in \mathcal{M}_{\alpha, n, \delta}^{2 s-2, \nu}[\ell], \mathcal{R}_{\ell} \in$ $\mathcal{R}_{\alpha, n}^{2 s, \nu}[\ell]$ such that

$$
\begin{aligned}
\sum_{\ell=0}^{n} \operatorname{Re} i\left\langle C_{\ell}(\underbrace{\bar{u}, \ldots, \bar{u}}_{\ell}, \underbrace{u, \ldots, u}_{n-\ell}) u, \Lambda_{\alpha, m}^{2 s} u\right\rangle= & \sum_{\ell=0}^{n} \operatorname{Re} i\left\langle\mathcal{M}_{\ell}(\underbrace{\bar{u}, \ldots, \bar{u}}_{\ell}, \underbrace{u, \ldots, u}_{n-\ell}) u, u\right\rangle \\
& +\sum_{\ell=0}^{n} \operatorname{Re} i\left\langle\mathcal{R}_{\ell}(\underbrace{\bar{u}, \ldots, \bar{u}}_{\ell}, \underbrace{u, \ldots, u}_{n-\ell}) u, u\right\rangle
\end{aligned}
$$

LEMmA 4.3. - For each integer $\ell \in[0, n]$, there are $\widehat{\mathcal{M}}_{\ell} \in \mathcal{M}_{\alpha, n, \delta}^{2 s-1, \nu}[\widehat{\ell]}$ and $\widehat{\mathcal{R}}_{\ell} \in \mathcal{R}_{\alpha, n}^{2 s, \nu}[\widehat{\ell]}$ such that 


$$
\begin{aligned}
\sum_{\ell=0}^{n} \operatorname{Re} i\left\langle C_{\ell}(\underbrace{\bar{u}, \ldots, \bar{u}}_{\ell}, \underbrace{u, \ldots, u}_{n-\ell}) \bar{u}, \Lambda_{\alpha, m}^{2 s} u\right\rangle= & \sum_{\ell=0}^{n} \operatorname{Re} i\left\langle\widehat{\mathcal{M}}_{\ell}(\underbrace{\bar{u}, \ldots, \bar{u}}_{\ell}, \underbrace{u, \ldots, u}_{n-\ell}) \bar{u}, u\right\rangle \\
& +\sum_{\ell=0}^{n} \operatorname{Re} i\left\langle\widehat{\mathcal{R}}_{\ell}(\underbrace{\bar{u}, \ldots, \bar{u}}_{\ell}, \underbrace{u, \ldots, u}_{n-\ell}) \bar{u}, u\right\rangle
\end{aligned}
$$

4.2. Analysis of the first term, proof of Lemma 4.2. - We explain how to handle the right side of (30). We force the apparition of multilinear integrals :

$$
\begin{aligned}
& C_{\ell}(\underbrace{\bar{u}, \ldots, \bar{u}}_{\ell}, \underbrace{u, \ldots, u}_{n-\ell}) f=\sum_{k_{0} \geq 1}\left\langle C_{\ell}(\underbrace{\bar{u}, \ldots, \bar{u}}_{\ell}, \underbrace{u, \ldots, u}_{n-\ell} f, \phi_{\alpha, k_{0}}\rangle \phi_{\alpha, k_{0}}\right. \\
= & \frac{1}{2^{n+1}}\left(\begin{array}{c}
n \\
\ell
\end{array}\right) \sum_{(\mathbb{N} \backslash\{0\})^{n+2}}\left\{b\left(k_{1}, \ldots, k_{n+1}\right)\left[\int_{\mathbb{R}}^{n} \prod_{j=0}^{n+1} \phi_{\alpha, k_{j}}(x) d x\right] \phi_{\alpha, k_{0}}\right. \\
& {\left.\left[\prod_{j=1}^{\ell}\left\langle\Lambda_{\alpha, m}^{-1} \bar{u}, \phi_{\alpha, k_{j}}\right\rangle\right]\left[\prod_{j=\ell+1}^{n}\left\langle\Lambda_{\alpha, m}^{-1} u, \phi_{\alpha, k_{j}}\right\rangle\right]\left\langle\Lambda_{\alpha, m}^{-1} f, \phi_{\alpha, k_{n+1}}\right\rangle\right\} . }
\end{aligned}
$$

Using the equality $\left\langle\Lambda_{\alpha, m}^{-1} u, \phi_{\alpha, k_{j}}\right\rangle=\left(\lambda_{\alpha, k_{j}}^{2}+m^{2}\right)^{-1 / 2}\left\langle u, \phi_{\alpha, k_{j}}\right\rangle$ allows us to change $b$ for a real map $b^{\prime}$ such that

$$
\begin{aligned}
& C_{\ell}(\underbrace{\bar{u}, \ldots, \bar{u}}_{\ell}, \underbrace{u, \ldots, u}_{n-\ell}) u \\
&= \sum_{(\mathbb{N} \backslash\{0\})^{n+2}}\left\{b^{\prime}\left(k_{0}, \ldots, k_{n+1}\right)\left[\prod_{j=1}^{\ell}\left\langle\bar{u}, \phi_{\alpha, k_{j}}\right\rangle\right]\left[\prod_{j=\ell+1}^{n+1}\left\langle u, \phi_{\alpha, k_{j}}\right\rangle\right] \phi_{\alpha, k_{0}}\right\} \\
& b^{\prime}\left(k_{0}, \ldots, k_{n+1}\right):=\frac{1}{2^{n+1}}\left(\begin{array}{c}
n \\
\ell
\end{array}\right) \frac{b\left(k_{1}, \ldots, k_{n+1}\right)}{\prod_{j=1}^{n+1} \sqrt{\lambda_{\alpha, k_{j}}^{2}+m^{2}}}\left|\int_{\mathbb{R}}^{n+1} \prod_{j=0}^{n+1} \phi_{\alpha, k_{j}}(x) d x\right| .
\end{aligned}
$$

Notice that since $b$ is bounded, one has

$$
\left|b^{\prime}\left(k_{0}, \ldots, k_{n+1}\right)\right| \leq \frac{C}{k_{1}^{\alpha^{\prime}} \ldots k_{n+1}^{\alpha^{\prime}}}\left|\int_{\mathbb{R}}^{n+1} \prod_{j=0} \phi_{\alpha, k_{j}}(x) d x\right| \leq \frac{C}{k_{n+1}^{\alpha^{\prime}}} \mid \int_{\mathbb{R}}^{n+1} \prod_{j=0}^{+1} \phi_{\alpha, k_{j}}(x) d x .
$$

To avoid a repetition of complicated terms, we will use the following notation

$$
C_{\ell}(\underbrace{\bar{u}, \ldots, \bar{u}}_{\ell}, \underbrace{u, \ldots, u}_{n-\ell})=\mathcal{F}\left(b^{\prime}\left(k_{0}, \ldots, k_{n+1}\right)\right) .
$$

We make the following decomposition

$$
b^{\prime}\left(k_{0}, \ldots, k_{n+1}\right)=b_{11}^{\prime}\left(k_{0}, \ldots, k_{n+1}\right)+b_{12}^{\prime}\left(k_{0}, \ldots, k_{n+1}\right)
$$


where $b_{11}^{\prime}\left(k_{0}, \ldots, k_{n+1}\right)$ carries $\left(k_{0}, \ldots, k_{n+1}\right)$ such that

$$
\delta \leq \frac{k_{n+1}}{k_{0}} \leq \frac{1}{\delta}, \quad \max \left(k_{1}, \ldots, k_{n}\right) \leq \delta^{2} k_{n+1} .
$$

The term $b_{12}^{\prime}\left(k_{0}, \ldots, k_{n+1}\right)$ carries others terms. This decomposition allows us to write

$$
\begin{gathered}
C_{\ell}(\underbrace{\bar{u}, \ldots, \bar{u}}_{\ell}, \underbrace{u, \ldots, u}_{n-\ell})=C_{\ell, 11}(\underbrace{\bar{u}, \ldots, \bar{u}}_{\ell}, \underbrace{u, \ldots, u}_{n-\ell})+C_{\ell, 12}(\underbrace{\bar{u}, \ldots, \bar{u}}_{\ell}, \underbrace{u, \ldots, u}_{n-\ell}) \\
C_{\ell, 11}(\underbrace{\bar{u}, \ldots, \bar{u}}_{\ell}, \underbrace{u, \ldots, u}_{n-\ell}):=\mathcal{F}\left(b_{11}^{\prime}\left(k_{0}, \ldots, k_{n+1}\right)\right) \\
C_{\ell, 12}(\underbrace{\bar{u}, \ldots, \bar{u}}_{\ell}, \underbrace{u, \ldots, u}_{n-\ell}):=\mathcal{F}\left(b_{12}^{\prime}\left(k_{0}, \ldots, k_{n+1}\right)\right) .
\end{gathered}
$$

The term $C_{\ell, 11}$ will contribute in $\mathcal{M}_{\alpha, n, \delta}^{2 s-2, \nu}[\ell]$ whereas the term $C_{\ell, 12}$, which must be seen as a rest, will contribute in $\mathcal{R}_{\alpha, n}^{2 s, \nu}$.

4.2.1. The term $C_{\ell, 11}$ and the commutator trick. - Remark the two relations

$\begin{aligned} \operatorname{Re} i\left\langle C_{\ell, 11}(\underbrace{\bar{u}, \ldots, \bar{u}}_{\ell}, \underbrace{u, \ldots, u}_{n-\ell}) u, \Lambda_{\alpha, m}^{2 s} u\right\rangle & =\operatorname{Re} i\left\langle\Lambda_{\alpha, m}^{2 s} C_{\ell, 11}(\underbrace{\bar{u}, \ldots, \bar{u}}_{\ell}, \underbrace{u, \ldots, u}_{n-\ell}) u, u\right\rangle \\ & =-\operatorname{Re} i\left\langle C_{\ell, 11}(\underbrace{\bar{u}, \ldots, \bar{u}}_{\ell}, \underbrace{u, \ldots, u}_{n-\ell})^{\star} \Lambda_{\alpha, m}^{2 s} u, u\right\rangle .\end{aligned}$

Introducing the sum it comes

$$
\begin{aligned}
& 2 \operatorname{Re} i\left\langle\Lambda_{\alpha, m}^{s} C_{\ell, 11}(\underbrace{\bar{u}, \ldots, \bar{u}}_{\ell}, \underbrace{u, \ldots, u}_{n-\ell}) u, \Lambda_{\alpha, m}^{s} u\right\rangle \\
& =\operatorname{Re} i\left\langle\Lambda_{\alpha, m}^{2 s} C_{\ell, 11}(\underbrace{\bar{u}, \ldots, \bar{u}}_{\ell}, \underbrace{u, \ldots, u}_{n-\ell}) u, u\right\rangle-i\left\langle C_{\ell, 11}(\underbrace{\bar{u}, \ldots, \bar{u}}_{\ell}, \underbrace{u, \ldots, u}_{n-\ell})^{\star} \Lambda_{\alpha, m}^{2 s} u, u\right\rangle \\
& =\operatorname{Re} i\left\langle C_{\ell, 11}(\underbrace{\bar{u}, \ldots, \bar{u}}_{\ell}, \underbrace{u, \ldots, u}_{n-\ell}) \Lambda_{\alpha, m}^{2 s} u-C_{\ell, 11}(\underbrace{\bar{u}, \ldots, \bar{u}}_{\ell}, \underbrace{u, \ldots, u}_{n-\ell})^{\star} \Lambda_{\alpha, m}^{2 s} u, u\right\rangle \\
& +\operatorname{Re} i\left\langle\left[\Lambda_{\alpha, m}^{2 s}, C_{\ell, 11}(\underbrace{\bar{u}, \ldots, \bar{u}}_{\ell}, \underbrace{u, \ldots, u}_{n-\ell})\right] u, u\right\rangle \text {. }
\end{aligned}
$$

The last term involves the commutator of $\Lambda_{\alpha, m}^{2 s}$ and $C_{\ell, 11}$. This is the main trick which allows a gain of regularity, that is the operator $\mathcal{M}_{\ell}$ will belong to $\mathcal{M}_{\alpha, n, \delta}^{2 s-2, \nu}$ instead of $\mathcal{M}_{\alpha, n, \delta}^{2 s-1, \nu}$. To our knowledge, this trick has been used for the first time in [Del09]. Let us compute the commutator $\left[\Lambda_{\alpha, m}^{2 s}, C_{\ell, 11}(\underbrace{\bar{u}, \ldots, \bar{u}}_{\ell}, \underbrace{u, \ldots, u}_{n-\ell})\right] u$ 
with the help of the notation (33). We see that

$$
\begin{aligned}
& \Lambda_{\alpha, m}^{2 s} C_{\ell, 11}(\underbrace{\bar{u}, \ldots, \bar{u}}_{\ell}, \underbrace{u, \ldots, u}_{n-\ell})=\mathcal{F}\left(\left(\lambda_{\alpha, k_{0}}^{2}+m^{2}\right)^{s} b_{11}^{\prime}\left(k_{0}, \ldots, k_{n+1}\right)\right) \\
& C_{\ell, 11}(\underbrace{\bar{u}, \ldots, \bar{u}}_{\ell}, \underbrace{u, \ldots, u}_{n-\ell}) \Lambda_{\alpha, m}^{2 s}=\mathcal{F}\left(\left(\lambda_{\alpha, k_{n+1}}^{2}+m^{2}\right)^{s} b_{11}^{\prime}\left(k_{0}, \ldots, k_{n+1}\right)\right) .
\end{aligned}
$$

Hence, the commutator $\left[\Lambda_{\alpha, m}^{2 s}, C_{\ell, 11}(\underbrace{\bar{u}, \ldots, \bar{u}}_{\ell}, \underbrace{u, \ldots, u}_{n-\ell})\right] u$ is nothing else than

$$
\mathcal{F}\left(b_{11}^{\prime}\left(k_{0}, \ldots, k_{n+1}\right)\left[\left(\lambda_{\alpha, k_{0}}^{2}+m^{2}\right)^{s}-\left(\lambda_{\alpha, k_{n+1}}^{2}+m^{2}\right)^{s}\right]\right) .
$$

Remember that in the support of $b_{11}$, one has $\delta \leq \frac{k_{0}}{k_{n+1}} \leq \frac{1}{\delta}$. Consequently, once $m$ is fixed, one has

$$
\forall k \geq 1 \quad\left(\lambda_{\alpha, k}^{2}+m^{2}\right) \leq\left(1+\frac{m^{2}}{\lambda_{\alpha, k}^{2}}\right) \lambda_{\alpha, k}^{2} \leq\left(1+\frac{m^{2}}{\lambda_{\alpha, 1}^{2}}\right) \lambda_{\alpha, k}^{2} \leq C_{m, \alpha} \lambda_{\alpha, k}^{2}
$$

and so

$$
\begin{aligned}
\left|\left(\lambda_{\alpha, k_{0}}^{2}+m^{2}\right)^{s}-\left(\lambda_{\alpha, k_{n+1}}^{2}+m^{2}\right)^{s}\right| & \leq\left|\lambda_{\alpha, k_{0}}-\lambda_{\alpha, k_{n+1}}\right| \times C(m, \alpha, \delta, s) \lambda_{\alpha, k_{0}}^{2 s-1} \\
& \leq C(m, \alpha, \delta, s)\left|k_{0}^{\alpha^{\prime}}-k_{n+1}^{\alpha^{\prime}}\right| k_{0}^{(2 s-1) \alpha^{\prime}} .
\end{aligned}
$$

Using (32) and Corollary 2.6 ensure that

$$
b_{11}^{\prime}\left(k_{0}, \ldots, k_{n+1}\right)\left[\left(\lambda_{\alpha, k_{0}}^{2}+m^{2}\right)^{s}-\left(\lambda_{\alpha, k_{n+1}}^{2}+m^{2}\right)^{s}\right]
$$

is bounded, for each $N>0$, by

$$
C_{N}\left|k_{0}^{\alpha^{\prime}}-k_{n+1}^{\alpha^{\prime}}\right| k_{0}^{(2 s-2) \alpha^{\prime}} \max \left(k_{1}, \ldots, k_{n}\right)^{\alpha^{\prime} \nu}\left(\frac{\max \left(k_{1}, \ldots, k_{n}\right)^{\alpha^{\prime}}}{\max \left(k_{1}, \ldots, k_{n}\right)^{\alpha^{\prime}}+\left|k_{0}^{\alpha^{\prime}}-k_{n+1}^{\alpha^{\prime}}\right|}\right)^{N}
$$

which is obviously less than

$$
C_{N} k_{0}^{(2 s-2) \alpha^{\prime}} \max \left(k_{1}, \ldots, k_{n}\right)^{\alpha^{\prime} \nu+1}\left(\frac{\max \left(k_{1}, \ldots, k_{n}\right)^{\alpha^{\prime}}}{\max \left(k_{1}, \ldots, k_{n}\right)^{\alpha^{\prime}}+\left|k_{0}^{\alpha^{\prime}}-k_{n+1}^{\alpha^{\prime}}\right|}\right)^{N-1}
$$

Let us define $\mathcal{M}_{\ell, 11 a}\left(u_{1}, \ldots, u_{n}\right) u_{n+1}$ by

$\sum_{(\mathbb{N} \backslash\{0\})^{n+2} \backslash \Omega_{n+2}(\ell)}\left\{b_{11}^{\prime}\left[\left(\lambda_{\alpha, k_{0}}^{2}+m^{2}\right)^{s}-\left(\lambda_{\alpha, k_{n+1}}^{2}+m^{2}\right)^{s}\right]\left[\prod_{j=1}^{n+1}\left\langle u_{j}, \phi_{\alpha, k_{j}}\right\rangle\right] \phi_{\alpha, k_{0}}\right\}$

where $\Omega_{n+2}(\ell)$ is defined in (17). This construction leads to

$\operatorname{Re} i\left\langle\left[\Lambda_{\alpha, m}^{2 s}, C_{\ell, 11}(\underbrace{\bar{u}, \ldots, \bar{u}}_{\ell}, \underbrace{u, \ldots, u}_{n-\ell})\right] u, u\right\rangle=\operatorname{Re} i\left\langle M_{\ell, 11 a}(\underbrace{\bar{u}, \ldots, \bar{u}}_{\ell}, \underbrace{u, \ldots, u}_{n-\ell}) u, u\right\rangle$ 
$+\operatorname{Re} i \sum_{\Omega_{n+2}(\ell)} b_{11}^{\prime}\left[\left(\lambda_{\alpha, k_{0}}^{2}+m^{2}\right)^{s}-\left(\lambda_{\alpha, k_{n+1}}^{2}+m^{2}\right)^{s}\right] \prod_{j=0}^{\ell}\left\langle\bar{u}, \phi_{\alpha, k_{j}}\right\rangle \prod_{j=\ell+1}^{n+1}\left\langle u, \phi_{\alpha, k_{j}}\right\rangle$.

The term $j=0$ comes from the scalar product by $u$. By symmetry, (37) is the real part of an imaginary number, so is clearly zero. Estimates (36) mean that $\mathcal{M}_{\ell, 11 a}$ belongs to $\mathcal{M}_{\alpha, n, \delta}^{2 s-2, \nu}[\ell]$. We have finished with the commutator term.

Looking at (35), we have now to consider

$\operatorname{Re} i\left\langle C_{\ell, 11}(\underbrace{\bar{u}, \ldots, \bar{u}}_{\ell}, \underbrace{u, \ldots, u}_{n-\ell}) \Lambda_{\alpha, m}^{2 s} u-C_{\ell, 11}(\underbrace{\bar{u}, \ldots, \bar{u}}_{\ell}, \underbrace{u, \ldots, u}_{n-\ell})^{\star} \Lambda_{\alpha, m}^{2 s} u, u\right\rangle$.

Remember we have

$$
\begin{aligned}
& C_{\ell, 11}(\underbrace{\bar{u}, \ldots, \bar{u}}_{\ell}, \underbrace{u, \ldots, u}_{n-\ell}) f \\
= & \sum_{(\mathbb{N} \backslash\{0\})^{n+2}} b_{11}^{\prime}\left[\prod_{j=1}^{\ell}\left\langle\bar{u}, \phi_{\alpha, k_{\ell}}\right\rangle\right]\left[\prod_{j=\ell+1}^{n}\left\langle u, \phi_{\alpha, k_{j}}\right\rangle\right]\left\langle f, \phi_{\alpha, k_{n+1}}\right\rangle \phi_{\alpha, k_{0}} .
\end{aligned}
$$

So, the computation of the adjoint operator is just given by permuting $\phi_{\alpha, k_{n+1}}$ and $\phi_{\alpha, k_{0}}$ :

$$
\begin{aligned}
& C_{\ell, 11}(\underbrace{\bar{u}, \ldots, \bar{u}}_{\ell}, \underbrace{u, \ldots, u}_{n-\ell})^{\star} f \\
= & \sum_{(\mathbb{N} \backslash\{0\})^{n+2}} b_{11}^{\prime}\left[\prod_{j=1}^{\ell}\left\langle\bar{u}, \phi_{\alpha, k_{\ell}}\right\rangle\right]\left[\prod_{j=\ell+1}^{n}\left\langle u, \phi_{\alpha, k_{j}}\right\rangle\right]\left\langle f, \phi_{\alpha_{k_{0}}}\right\rangle \phi_{\alpha, k_{n+1}} \\
= & \sum b_{11}^{\prime}\left(k_{n+1}, k_{1}, \ldots, k_{n}, k_{0}\right) \prod_{j=1}^{n}\left\langle\bar{u}, \phi_{\alpha, k_{\ell}}\right\rangle \prod_{j=\ell+1}^{n}\left\langle u, \phi_{\alpha, k_{j}}\right\rangle\left\langle f, \phi_{\alpha, k_{n+1}}\right\rangle \phi_{\alpha, k_{0}} .
\end{aligned}
$$

Then it follows that (38) is just

$$
\begin{gathered}
\operatorname{Re} i \sum_{(\mathbb{N} \backslash\{0\})^{n+2}}\left[b_{11}^{\prime}-\widetilde{b_{11}^{\prime}}\right]\left(\lambda_{\alpha, k_{n+1}}^{2}+m^{2}\right)^{s} \prod_{j=0}^{\ell}\left\langle\bar{u}, \phi_{\alpha, k_{\ell}}\right\rangle \prod_{j=\ell+1}^{n+1}\left\langle u, \phi_{\alpha, k_{j}}\right\rangle, \\
\widetilde{b_{11}^{\prime}}\left(k_{0}, k_{1}, \ldots, k_{n}, k_{n+1}\right):=b_{11}^{\prime}\left(k_{n+1}, k_{1}, \ldots, k_{n}, k_{0}\right) .
\end{gathered}
$$

But $b_{11}^{\prime}-\widetilde{b_{11}^{\prime}}$ is also

$$
\left[\frac{b^{\prime}\left(k_{1}, \ldots, k_{n+1}\right)}{\prod_{j=1}^{n+1} \sqrt{\lambda_{\alpha, k_{j}}^{2}+m^{2}}}-\frac{b^{\prime}\left(k_{1}, \ldots, k_{n}, k_{0}\right)}{\prod_{j=0}^{n} \sqrt{\lambda_{\alpha, k_{j}}^{2}+m^{2}}}\right] \int_{\mathbb{R}}^{n+1} \prod_{j=0}^{n+1} \phi_{\alpha, k_{j}}(x) d x .
$$

As we are in the zone of $(\mathbb{N} \backslash\{0\})^{n+2}$ ruled by the condition (34), if $\delta$ is less than 1 then $\max \left(k_{1}, \ldots, k_{n}\right)<\min \left(k_{0}, k_{n+1}\right)$ obviously holds. The definition of $b$ forces the two numbers $b^{\prime}\left(k_{1}, \ldots, k_{n+1}\right)$ and $b^{\prime}\left(k_{1}, \ldots, k_{n}, k_{0}\right)$ to take the 
same value (which is moreover bounded with respect to $k_{0}, \ldots, k_{n+1}$ ). We understand that (39) is bounded by

$$
C\left|\frac{1}{\sqrt{\lambda_{\alpha, k_{0}}^{2}+m^{2}}}-\frac{1}{\sqrt{\lambda_{\alpha, k_{n+1}}^{2}+m^{2}}}\right| \times\left|\int_{\mathbb{R}} \prod_{j=0}^{n+1} \phi_{\alpha, k_{j}}(x) d x\right| .
$$

With the help of Corollary (2.6) and

$$
\lambda_{\alpha, k_{n+1}}^{2 s}\left(\frac{1}{\sqrt{\lambda_{\alpha, k_{0}}^{2}+m^{2}}}-\frac{1}{\sqrt{\lambda_{\alpha, k_{n+1}}^{2}+m^{2}}}\right) \leq C\left|\lambda_{\alpha, k_{0}}-\lambda_{\alpha, k_{n+1}}\right| \lambda_{\alpha, k_{n+1}}^{2 s-2}
$$

we can use the same estimates that for the commutator term to obtain an adequate operator in $\mathcal{M}_{\alpha, n, \delta}^{2 s-2, \nu}[\ell]$. The term $C_{\ell, 11}$ is completely done.

4.2.2. The term $C_{\ell, 12}$ seen as a rest. - Let us finish the proof of Lemma 4.2. We have to introduce an operator $\mathcal{R}_{\ell} \in \mathcal{R}_{\alpha, n}^{2 s, \nu}[\ell]$ such that

$$
\operatorname{Re} i\left\langle C_{\ell, 12}(\underbrace{\bar{u}, \ldots, \bar{u}}_{\ell}, \underbrace{u, \ldots, u}_{n-\ell}) u, \Lambda_{\alpha, m}^{2 s} u\right)=\operatorname{Re} i\left\langle\mathcal{R}_{\ell}(\underbrace{\bar{u}, \ldots, \bar{u}}_{\ell}, \underbrace{u, \ldots, u}_{n-\ell}) u, u\right\rangle .
$$

Following the same idea as above, it is clear that a convenient operator is given by

$$
\mathcal{R}_{\ell}\left(u_{1}, \ldots, u_{n}\right) u_{n+1}=\sum_{\Omega_{n+2}(\ell)^{c}}\left\{b_{12}^{\prime}\left[\prod_{j=1}^{n+1}\left\langle u_{j}, \phi_{\alpha, k_{j}}\right\rangle\right]\left(\lambda_{\alpha, k_{0}}^{2}+m^{2}\right)^{s} \phi_{\alpha, k_{0}}\right\} .
$$

Here $\Omega_{n+2}(\ell)^{c}$ is by definition $(\mathbb{N} \backslash\{0\})^{n+2} \backslash \Omega_{n+2}(\ell)$. Remember now the definition of $b_{12}^{\prime}$ and $b^{\prime}$ (see (31)). Point ii) of Corollary 2.6 and the obvious inequality $\left|\lambda_{\alpha, k_{0}}^{2}+m^{2}\right|^{s} \leq C k_{0}^{2 \alpha^{\prime} s}$ ensure that $\mathcal{R}_{\ell} \in \mathcal{R}_{\alpha, n}^{2 s, \nu}$ for some $\nu>0$.

4.3. Analysis of the second term, proof of Lemma 4.3. - The proof is simpler than the one of Lemma 4.2. We also make a decomposition of $C_{\ell}$ with the help of the notation (33) :

$$
\begin{aligned}
& b_{21}^{\prime}\left(k_{0}, \ldots, k_{n+1}\right)=b^{\prime}\left(k_{0}, \ldots, k_{n+1}\right) \mathbf{1}_{\delta \leq \frac{k_{n+1}}{k_{0}} \leq \frac{1}{\delta}} \\
& b_{22}^{\prime}\left(k_{0}, \ldots, k_{n+1}\right)=b^{\prime}\left(k_{0}, \ldots, k_{n+1}\right) \mathbf{1}_{\frac{k_{n+1}}{k_{0}} \notin\left[\delta, \frac{1}{\delta}\right]} .
\end{aligned}
$$

Thus we define

$$
\begin{aligned}
& C_{\ell, 21}(\underbrace{\bar{u}, \ldots, \bar{u}}_{\ell}, \underbrace{u, \ldots, u}_{n-\ell})=\mathcal{F}\left(b_{21}^{\prime}\left(k_{0}, \ldots, k_{n+1}\right)\right) \\
& C_{\ell, 22}(\underbrace{\bar{u}, \ldots, \bar{u}}_{\ell}, \underbrace{u, \ldots, u}_{n-\ell})=\mathcal{F}\left(b_{22}^{\prime}\left(k_{0}, \ldots, k_{n+1}\right)\right) .
\end{aligned}
$$


In the conclusion of Lemma 4.3, the operators $C_{\ell, 21}$ and $C_{\ell, 22}$ will respectively contribute to $\mathcal{M}_{n, \alpha, \delta}^{2 s-1, \nu}\left[\widehat{\ell]}\right.$ and $\mathcal{R}_{n, \alpha}^{2 s, \nu}$ for some $\nu>0$. Let us begin with $C_{\ell, 21}$. We define $\widehat{M}_{\ell}$ to be

$$
\widehat{M}_{\ell}\left(u_{1}, \ldots, u_{n}\right) u_{n+1}=\sum_{\Omega_{n+2}(\widehat{\ell})^{c}} b_{21}^{\prime}\left[\prod_{j=1}^{n+1}\left\langle u_{j}, \phi_{\alpha, k_{j}}\right\rangle\right]\left(\lambda_{\alpha, k_{0}}^{2}+m^{2}\right)^{s} \phi_{\alpha, k_{0}}
$$

where $\Omega_{n+2}(\widehat{\ell})$ is defined in (17) and $\Omega_{n+2}(\widehat{\ell})$ is its complementary subset. Consequently, it is obvious that for all $u \in \mathcal{S}(\mathbb{R})$ we have

$$
\begin{aligned}
& \operatorname{Re} i\left\langle C_{\ell, 21}(\underbrace{\bar{u}, \ldots, \bar{u}}_{\ell}, \underbrace{u, \ldots, u}_{n-\ell}) \bar{u}, u\right\rangle= \\
& \operatorname{Re} i\left(\left\langle\widehat{M}_{\ell}(\underbrace{\bar{u}, \ldots, \bar{u}}_{\ell}, \underbrace{u, \ldots, u}_{n-\ell}) \bar{u}, u\right\rangle+\sum_{\Omega_{n+2}(\widehat{\ell})}\left\{b_{21}^{\prime}\left(k_{0}, \ldots, k_{n+1}\right)\left[\prod_{j=0}^{\ell}\left\langle\bar{u}, \phi_{\alpha, k_{j}}\right\rangle\right]\right.\right. \\
& \left.\left.\times\left\langle\bar{u}, \phi_{\alpha, k_{n+1}}\right\rangle\left[\prod_{j=\ell+1}^{n}\left\langle u, \phi_{\alpha, k_{j}}\right\rangle\right]\left(\lambda_{\alpha, k_{0}}^{2}+m^{2}\right)^{s} \phi_{\alpha, k_{0}}\right\}\right) .
\end{aligned}
$$

As already used, the symmetry of the last sum forces it to vanish. Thus, we just have to check that $\mathcal{M}_{\ell} \in \mathcal{M}_{n, \alpha, \delta}^{2 s-1, \nu}[\widehat{\ell}]$ for some $\nu>0$. Remember one has

$$
\begin{aligned}
\left(\lambda_{\alpha, k_{0}}^{2}+m^{2}\right)^{s}\left|b_{21}^{\prime}\left(k_{0}, \ldots, k_{n+1}\right)\right| & \leq \frac{C k_{0}^{2 \alpha^{\prime} s}}{\prod_{j=1}^{n+1} k_{j}^{\alpha^{\prime}}}\left|\int_{\mathbb{R}}^{n+1} \prod_{j=0}^{n+1} \phi_{\alpha, k_{j}}(x) d x\right| \mathbf{1}_{\delta \leq \frac{k_{n+1}}{k_{0}} \leq \frac{1}{\delta}} \\
& \leq C k_{0}^{\alpha^{\prime}(2 s-1)}\left|\int_{\mathbb{R}}^{n+1} \prod_{j=0} \phi_{\alpha, k_{j}}(x) d x\right| \mathbf{1}_{\delta \leq \frac{k_{n+1}}{k_{0}} \leq \frac{1}{\delta}}
\end{aligned}
$$

Notice now that $b_{21}^{\prime}$ has a support included in $\left\{\max \left(k_{1}, \ldots, k_{n}\right) \leq k_{n+1}\right\}$. Point i) of Corollary 2.6 proves $\mathcal{M}_{\ell} \in \mathcal{M}_{n, \alpha, \delta}^{2 s-1, \nu}[\widehat{\ell]}$ for some $\nu>0$.

The same argumentation works with $C_{\ell, 22}$ and produce an adequate operator $\mathcal{R}_{\ell} \in \mathcal{R}_{n, \alpha}^{2 s, \nu}[\widehat{\ell}]$. Indeed we just have to prove that there is some $\nu>0$ such that for all $N>0$ one has

$$
\left(\lambda_{\alpha, k_{0}}^{2}+m^{2}\right)^{s}\left|b_{22}^{\prime}\left(k_{0}, \ldots, k_{n+1}\right)\right| \leq C k_{0}^{2 \alpha^{\prime} s} \frac{\max _{2}\left(k_{1}^{\alpha^{\prime}}, \ldots, k_{n+1}^{\alpha^{\prime}}\right)^{\nu+N}}{\left(k_{0}^{\alpha^{\prime}}+\cdots+k_{n+1}^{\alpha^{\prime}}\right)^{N}} .
$$

That is a consequence of Point ii) of Corollary 2.6.

\section{Elimination of terms}

5.1. Construction of new operators. - Let us begin by a lemma which makes rigorous heuristic derivations. 
LEMMA 5.1. - Let $s$ be an real number larger than 1 and $u \in \mathcal{C}^{0}\left(I, \mathcal{H}_{\alpha}^{s}\right)$ be a $\mathcal{H}_{\alpha}^{s}$ strong solution of $\dot{u}=i \Lambda_{\alpha, m} u-i w^{n+1}$ where $w=\Lambda_{\alpha, m}^{-1} \operatorname{Re}(u)$. Moreover we consider two bounded $\mathbb{R}$-multilinear forms $M$ and $B$ from $\left(\mathcal{H}_{\alpha}^{s}\right)^{n+2}$ to $\mathbb{C}$ such that for all $\phi \in \mathcal{H}_{\alpha}^{s+1}$

$$
\sum_{k=1}^{n+2} M(\underbrace{\phi, \ldots, \phi}_{k-1}, i \Lambda_{\alpha, m} \phi, \phi, \ldots, \phi)=B(\phi, \ldots, \phi) .
$$

Then one has the following derivative

$$
\frac{d}{d t} M(u, \ldots, u)=B(u, \ldots, u)+\sum_{k=1}^{n+2} M(\underbrace{u, \ldots, u}_{k-1},-i w^{n+1}, u, \ldots, u) .
$$

Proof. - The difficulty here is that $M\left(i \Lambda_{\alpha, m} u, u, \ldots, u\right)$ has no sense "a priori" if $u$ is a $\mathcal{H}_{\alpha}^{s}$ strong solution (because $i \Lambda_{\alpha, m} u \in \mathcal{H}_{\alpha}^{s-1}$ ). Of course the result is obvious if $u$ is $\mathcal{H}_{\alpha}^{s+1}$ strong solution because one has automatically $u \in$ $\mathcal{C}^{1}\left(I, \mathcal{H}_{\alpha}^{s}\right)$. To prove the lemma, we use a standard density argument. Consider a $\mathcal{H}_{\alpha}^{s}$ strong solution $u$, an integer $R>1$ and define

$$
u_{R, 0}(x)=\sum_{k=1}^{R}\left\langle u(0, \cdot), \phi_{\alpha, k}\right\rangle \phi_{\alpha, k} .
$$

Define now $u_{R}(t, x)$ the $\mathcal{H}_{\alpha}^{s+1}$ strong solution with initial datum $u_{R, 0} \in \mathcal{H}_{\alpha}^{s+1}$. Thus, the gain of regularity allows to write for all $t$

$$
\begin{gathered}
M\left(u_{R}, \ldots, u_{R}\right)-M\left(u_{R, 0}, \ldots, u_{R, 0}\right)=\int_{0}^{t} B\left(u_{R}(\tau), \ldots, u_{R}(\tau)\right) d \tau \\
+\int_{0}^{t} \sum_{k=1}^{n+2} M(\underbrace{u_{R}(\tau), \ldots, u_{R}(\tau)}_{k-1},-i \operatorname{Re}\left(\Lambda_{\alpha, m}^{-1} u_{R}(\tau)\right)^{n+1}, u_{R}(\tau), \ldots, u_{R}(\tau)) d \tau .
\end{gathered}
$$

Using twice Duhamel formula, we see that $u_{R}(t, \cdot)-u(t, \cdot)$ is

$$
e^{i t \Lambda_{\alpha, m}}\left(u_{R, 0}-u_{0}\right)-i \int_{0}^{t} e^{i(t-\tau) \Lambda_{\alpha, m}}\left[\operatorname{Re}\left(u(\tau, \cdot)^{n+1}\right)-\operatorname{Re}\left(u_{R}(\tau, \cdot)^{n+1}\right)\right] d \tau .
$$

Hence, we have

$$
\left\|u_{R}-u\right\|_{\mathcal{H}_{\alpha}^{s}} \leq\left\|u_{R, 0}-u_{0}\right\|_{\mathcal{H}_{\alpha}^{s}}+C\left(\|u\|_{L_{t}^{\infty} H_{\alpha}^{s}},\left\|u_{R}\right\|_{L_{t}^{\infty} H_{\alpha}^{s}}\right) \int_{0}^{t}\left\|u-u_{R}\right\|_{\mathcal{H}_{\alpha}^{s}} d \tau \text {. }
$$

Gronwall's inequality gives for any time $t$

$$
\lim _{R \rightarrow+\infty}\left\|u_{R}(t)-u(t)\right\|_{\mathcal{H}_{\alpha}^{s}}=0 .
$$

To conclude, let $R$ tend to infinity in (40) and (41). 
Let $\mathcal{M}_{\ell} \in \mathcal{M}_{\alpha, n, \delta}^{2 s-2, \nu}[\ell], \widehat{\mathcal{M}}_{\ell} \in \mathcal{M}_{\alpha, n, \delta}^{2 s-1, \nu}[\widehat{\ell}], \mathcal{R}_{\ell} \in \mathcal{R}_{n, \alpha}^{2 s, \nu}[\ell]$ and $\widehat{\mathcal{R}}_{\ell} \in \mathcal{R}_{n, \alpha}^{2 s, \nu}[\widehat{\ell}]$ be the operators introduced in Proposition 4.1.

Proposition 5.2. - We suppose that $\alpha \geq 4$ holds. For $s$ enough large and $\rho>0$, there are $\nu_{0}>0$ and multilinear operators

$$
\begin{array}{rlrl}
\underline{\mathcal{M}_{\ell}} & \in \mathcal{M}_{\alpha, n, \delta}^{2 s-1+\frac{2}{\alpha}+\rho, \nu+\nu_{0}}[\ell], \underline{\widehat{\mathcal{M}}_{\ell}} & \in \mathcal{M}_{\alpha, n, \delta}^{2 s-2, \nu+\nu_{0}}[\widehat{\ell]} \\
\underline{\mathcal{R}_{\ell}} & \in \mathcal{R}_{n, \alpha}^{2 s, \nu+\nu_{0}}[\ell], & \underline{\widehat{\mathcal{R}}_{\ell}} & \in \mathcal{R}_{n, \alpha}^{2 s-1, \nu+\nu_{0}}[\widehat{\ell]}
\end{array}
$$

such that for any $\mathcal{H}_{\alpha}^{s}$ strong solution $u$ of $\dot{u}=i \Lambda_{\alpha, m} u-i w^{n+1}$, one has

$$
\begin{aligned}
\frac{d}{d t}\left\langle\underline{\mathcal{M}_{\ell}}(\bar{u}, \ldots, \bar{u}, u, \ldots, u) u, u\right\rangle & =\left\langle\mathcal{M}_{\ell}(\bar{u}, \ldots, \bar{u}, u, \ldots, u) u, u\right\rangle+\mathcal{O}\left(\|u\|_{\mathcal{H}_{\alpha}^{s}}^{2 n+2}\right) \\
\frac{d}{d t}\left\langle\underline{\widehat{\mathcal{M}}_{\ell}}(\bar{u}, \ldots, \bar{u}, u, \ldots, u) \bar{u}, u\right\rangle & =\left\langle\widehat{\mathcal{M}}_{\ell}(\bar{u}, \ldots, \bar{u}, u, \ldots, u) \bar{u}, u\right\rangle+\mathcal{O}\left(\|u\|_{\mathcal{H}_{\alpha}^{s}}^{2 n+2}\right) \\
\frac{d}{d t}\left\langle\underline{\mathcal{R}_{\ell}}(\bar{u}, \ldots, \bar{u}, u, \ldots, u) u, u\right\rangle & =\left\langle\mathcal{R}_{\ell}(\bar{u}, \ldots, \bar{u}, u, \ldots, u) u, u\right\rangle+\mathcal{O}\left(\|u\|_{\mathcal{H}_{\alpha}^{s}}^{2 n+2}\right) \\
\frac{d}{d t} & \left.\underline{\widehat{\mathcal{R}}_{\ell}}(\underbrace{\bar{u}, \ldots, \bar{u}}_{\ell}, \underbrace{u, \ldots, u}_{n-\ell}) \bar{u}, u\right\rangle=\left\langle\widehat{\mathcal{R}}_{\ell}(\underbrace{\bar{u}, \ldots, \bar{u}}_{\ell}, \underbrace{u, \ldots, u}_{n-\ell}) \bar{u}, u\right\rangle+\mathcal{O}\left(\|u\|_{\mathcal{H}_{\alpha}^{s}}^{2 n+2}\right) .
\end{aligned}
$$

Proof. - The regularity $s$ is chosen to be enough large such that each space of multilinear operators have the $\mathcal{H}_{\alpha}^{s}$ boundedness property (see Proposition 3.2 with $\tau \leq 2 s-\frac{1}{\alpha}$ and Proposition 3.7 with $\tau=2 s$ ). Let us compute $\underline{\mathcal{M}_{\ell}}$, other terms are similar. Definition 3.1 allows us to write

$$
\left\langle\mathcal{M}_{\ell}\left(u_{1}, \ldots, u_{n}\right) u_{n+1}, u_{0}\right\rangle=\sum_{(\mathbb{N} \backslash\{0\})^{n+2} \backslash \Omega_{n+2}(\ell)} m_{\ell}\left(k_{0}, \ldots, k_{n+1}\right) \prod_{j=0}^{n+1}\left\langle u_{j}, \phi_{\alpha, k_{j}}\right\rangle
$$

where $\left|m_{\ell}\left(k_{0}, \ldots, k_{n+1}\right)\right|$ is bounded by

$$
C k_{0}^{\alpha^{\prime}(2 s-2)} \max \left(k_{1}^{\alpha^{\prime} \nu}, \ldots, k_{n}^{\alpha^{\prime} \nu}\right)\left(\frac{\max \left(k_{1}, \ldots, k_{n}\right)^{\alpha^{\prime}}}{\max \left(k_{1}, \ldots, k_{n}\right)^{\alpha^{\prime}}+\left|k_{0}^{\alpha^{\prime}}-k_{n+1}^{\alpha^{\prime}}\right|}\right)^{N}
$$

and that $m_{\ell}$ has support conditions $k_{n+1} \simeq k_{0}$ and $\max \left(k_{1}, \ldots, k_{n}\right) \leq k_{n+1}$. Now define the operator $\underline{\mathcal{M}_{\ell}}$ by the following expression.

$$
\left\langle\underline{\mathcal{M}_{\ell}}\left(u_{1}, \ldots, u_{n}\right) u_{n+1}, u_{0}\right\rangle=\sum_{\Omega_{n+2}(\ell)^{c}} \frac{m_{\ell}\left(k_{0}, \ldots, k_{n+1}\right)}{-i F_{m}^{\ell}\left(\lambda_{\alpha, k_{0}}, \ldots, \lambda_{\alpha, k_{n+1}}\right)} \prod_{j=0}^{n+1}\left\langle u_{j}, \phi_{\alpha, k_{j}}\right\rangle .
$$

First of all, we have to check that $\underline{\mathcal{M}_{\ell}}$ is a suitable operator. Indeed, from (18) and (43), we get for almost every $\bar{m}>0$

$$
\left|\frac{m_{\ell}\left(k_{0}, \ldots, k_{n+1}\right)}{i F_{m}^{\ell}\left(\lambda_{\alpha, k_{0}}, \ldots, \lambda_{\alpha, k_{n+1}}\right)}\right| \leq \frac{C k_{0}^{\alpha^{\prime}\left(2 s-1+\frac{2}{\alpha}+\rho\right)} \max \left(k_{1}, \ldots, k_{n}\right)^{\alpha^{\prime}(N+\nu)}}{\left(\max \left(k_{1}, \ldots, k_{n}\right)^{\alpha^{\prime}}+\left|k_{0}^{\alpha^{\prime}}-k_{n+1}^{\alpha^{\prime}}\right|\right)^{N-\nu_{0}}} .
$$


As $N$ is arbitrary, that proves $\mathcal{M}_{\ell}$ belongs to $\mathcal{M}_{\alpha, n, \delta}^{2 s-1+\frac{2}{\alpha}+\rho, \nu+\nu_{0}}[\ell]$. Therefore, as $\alpha \geq 4$ holds, one has for $\rho$ enough small

$$
2 s-1+\frac{2}{\alpha}+\rho<2 s-\frac{1}{\alpha} .
$$

Then, Proposition 3.2 ensures that $\underline{\mathcal{M}_{\ell}}$ is bounded from $\left(\mathcal{H}_{\alpha}^{s}\right)^{n}$ to $\mathcal{L}\left(\mathcal{H}_{\alpha}^{s}, \mathcal{H}_{\alpha}^{-s}\right)$. An easy computation gives

$$
\begin{aligned}
& -i\left\langle\underline{\mathcal{M}_{\ell}}(\underbrace{\bar{u}, \ldots, \bar{u}}_{\ell}, \underbrace{u, \ldots, u}_{n-\ell}) u, \Lambda_{\alpha, m} u\right\rangle \\
& -i \sum_{k=1}^{\ell}\left\langle\underline{\mathcal{M}_{\ell}}(\underbrace{\bar{u}, \ldots, \bar{u}}_{k-1}, \Lambda_{\alpha, m} \bar{u}, \underbrace{\bar{u}, \ldots, \bar{u}}_{\ell-k}, \underbrace{u, \ldots, u}_{n-\ell}) u, u\right\rangle \\
& \left.+i \sum_{k=\ell+1}^{n} \underline{\left\langle\mathcal{M}_{\ell}\right.}(\underbrace{\bar{u}, \ldots, \bar{u}}_{\ell}, \underbrace{u, \ldots, u}_{k-\ell-1}, \Lambda_{\alpha, m} u, \underbrace{u, \ldots, u}_{n-k}) u, u\right\rangle \\
& +i\left\langle\underline{\mathcal{M}_{\ell}}(\bar{u}, \ldots, \bar{u}, u, \ldots, u) \Lambda_{\alpha, m} u, u\right\rangle \\
& =\left\langle\mathcal{M}_{\ell}(\bar{u}, \ldots, \bar{u}, u, \ldots, u) u, u\right\rangle .
\end{aligned}
$$

Now we just have to use Lemma 5.1 with

$$
\begin{aligned}
M\left(u_{0}, \ldots, u_{n+1}\right) & =\left\langle\underline{\mathcal{M}_{\ell}}\left(\overline{u_{1}}, \ldots, \overline{u_{\ell}}, u_{\ell+1}, \ldots, u_{n}\right) u_{n}, u_{0}\right\rangle \\
B\left(u_{0}, \ldots, u_{n+1}\right) & =\left\langle\mathcal{M}_{\ell}\left(\overline{u_{1}}, \ldots, \overline{u_{\ell}}, u_{\ell+1}, \ldots, u_{n}\right) u_{n}, u_{0}\right\rangle .
\end{aligned}
$$

The rest $\mathcal{O}\left(\|u\|_{\mathcal{H}_{\alpha}^{s}}^{2 n+2}\right)$ comes from the boundedness of $M$ and and the inequality $\left\|w^{n+1}\right\|_{\mathcal{H}_{\alpha}^{s}} \leq C\|u\|_{\mathcal{H}_{\alpha}^{s}}^{n+1}$. We can treat other terms by the same strategy. Indeed we write :

$$
\begin{aligned}
\left\langle\widehat{\mathcal{M}}_{\ell}\left(u_{1}, \ldots, u_{n}\right) u_{n+1}, u_{0}\right\rangle & =\sum_{\Omega_{n+2}(\widehat{\ell})^{c}} \widehat{m}_{\ell}\left(k_{0}, \ldots, k_{n+1}\right) \prod_{j=0}^{n+1}\left\langle u_{j}, \phi_{\alpha, k_{j}}\right\rangle \\
\left\langle\underline{\widehat{\mathcal{M}}_{\ell}}\left(u_{1}, \ldots, u_{n}\right) u_{n+1}, u_{0}\right\rangle & :=\sum_{\Omega_{n+2}(\widehat{\ell})^{c}} \frac{\widehat{m}_{\ell}\left(k_{0}, \ldots, k_{n+1}\right)}{-i \widehat{F}_{m}^{\ell}\left(\lambda_{\alpha, k_{0}}, \ldots, \lambda_{\alpha, k_{n+1}}\right)} \prod_{j=0}^{n+1}\left\langle u_{j}, \phi_{\alpha, k_{j}}\right\rangle .
\end{aligned}
$$

We see that $\underline{\widehat{\mathcal{M}}_{\ell}}$ belongs to $\mathcal{M}_{\alpha, n, \delta}^{2 s-2, \nu}$ with the help of (20). We also write :

$$
\begin{aligned}
\left\langle\mathcal{R}_{\ell}\left(u_{1}, \ldots, u_{n}\right) u_{n+1}, u_{0}\right\rangle & =\sum_{\Omega_{n+2}(\ell)^{c}} r_{\ell}\left(k_{0}, \ldots, k_{n+1}\right) \prod_{j=0}^{n+1}\left\langle u_{j}, \phi_{\alpha, k_{j}}\right\rangle \\
\left\langle\underline{\mathcal{R}_{\ell}}\left(u_{1}, \ldots, u_{n}\right) u_{n+1}, u_{0}\right\rangle & :=\sum_{\Omega_{n+2}(\ell)^{c}} \frac{r_{\ell}\left(k_{0}, \ldots, k_{n+1}\right)}{-i F_{m}^{\ell}\left(\lambda_{\alpha, k_{0}}, \ldots, \lambda_{\alpha, k_{n+1}}\right)} \prod_{j=0}^{n+1}\left\langle u_{j}, \phi_{\alpha, k_{j}}\right\rangle .
\end{aligned}
$$


In a similar spirit, (19) implies that $\underline{\mathcal{R}_{\ell}} \in \mathcal{R}_{n, \alpha}^{2 s, \nu+\nu_{0}}$. Finally, we have

$$
\begin{aligned}
\left\langle\widehat{\mathcal{R}}_{\ell}\left(u_{1}, \ldots, u_{n}\right) u_{n+1}, u_{0}\right\rangle & =\sum_{\Omega_{n+2}(\widehat{\ell})^{c}} \widehat{r}_{\ell}\left(k_{0}, \ldots, k_{n+1}\right) \prod_{j=0}^{n+1}\left\langle u_{j}, \phi_{\alpha, k_{j}}\right\rangle \\
\left\langle\underline{\widehat{\mathcal{R}}_{\ell}}\left(u_{1}, \ldots, u_{n}\right) u_{n+1}, u_{0}\right\rangle:= & \sum_{\Omega_{n+2}(\widehat{\ell})^{c}} \frac{\widehat{r}_{\ell}\left(k_{0}, \ldots, k_{n+1}\right)}{-i \widehat{F}_{m}^{\ell}\left(\lambda_{\alpha, k_{0}}, \ldots, \lambda_{\alpha, k_{n+1}}\right)} \prod_{j=0}^{n+1}\left\langle u_{j}, \phi_{\alpha, k_{j}}\right\rangle .
\end{aligned}
$$

And we can use (21) to check that $\underline{\widehat{\mathcal{R}}_{\ell}} \in \mathcal{R}_{n, \alpha}^{2 s, \nu+\nu_{0}}$.

REMARK 5.3. - The line (44) is the only point where we need the condition $\alpha>3$, or equivalently $\alpha^{\prime}=\frac{\alpha}{1+\alpha}>\frac{3}{4}$. As we have $\lambda_{\alpha, k} \simeq k^{\alpha^{\prime}}$ and $\lambda_{\alpha, k}-\lambda_{\alpha, \ell} \simeq$ $k^{\alpha^{\prime}}-\ell^{\alpha^{\prime}}$, that means that in some sense the separation and the growth of the sequence $\left(\lambda_{\alpha, k}\right)_{k \geq 1}$ is enough important to ensure a complete normal form at the first step.

5.2. Conclusion if $\boldsymbol{\alpha} \geq \mathbf{4}$ holds. - We can now prove Theorem 1.6. For $s$ enough large, if $u$ is a $\mathcal{H}_{\alpha}^{s}$ strong solution of $\dot{u}=i \Lambda_{\alpha, m} u-i w^{n+1}$ then define

$$
\begin{aligned}
& M_{s}(u, \ldots, u) \\
:= & \operatorname{Re} i \sum_{\ell=0}^{n}\left[\left\langle\underline{\mathcal{M}_{\ell}}(\underbrace{\bar{u}, \ldots, \bar{u}}_{\ell}, \underbrace{u, \ldots, u}_{n-\ell}) u, u\right\rangle+\left\langle\underline{\widehat{\mathcal{M}}_{\ell}}(\underbrace{\bar{u}, \ldots, \bar{u}}_{\ell}, \underbrace{u, \ldots, u}_{n-\ell}) \bar{u}, u\right\rangle\right. \\
& \left.+\left\langle\underline{\mathcal{R}_{\ell}}(\underbrace{\bar{u}, \ldots, \bar{u}}_{\ell}, \underbrace{u, \ldots, u}_{n-\ell}) u, u\right\rangle+\left\langle\underline{\widehat{\mathcal{R}}_{\ell}}(\underbrace{\bar{u}, \ldots, \bar{u}}_{\ell}, \underbrace{u, \ldots, u}_{n-\ell}) \bar{u}, u\right\rangle\right] .
\end{aligned}
$$

Proposition 4.1 and Proposition 5.2 allow us to claim that $M_{s}$ has the $\mathcal{H}_{\alpha}^{s}$ boundedness property and

$$
\frac{d}{d t}\left[M_{s}(u, \ldots, u)-\Theta_{s}(u)\right]=\mathcal{O}\left(\|u\|_{\mathcal{H}_{\alpha}^{s}}^{2 n+2}\right) .
$$

As explained in part 1.2, this implies the local existence time can be improved to $\varepsilon^{2-2-2 n}=\varepsilon^{-2 n}$ if the initial data $u(0)$ has a small $\mathcal{H}_{\alpha}^{s}$-norm of order $\varepsilon \ll 1$. Theorem 1.6 is proved, and so is Theorem 1.2 .

5.3. What if $\boldsymbol{\alpha} \in\{\mathbf{2}, \mathbf{3}\}$ holds. - We have to modify the part of Proposition 5.2 which involves $\mathcal{M}_{\ell}$. Let us consider a function $\psi:(0,1) \rightarrow \mathbb{R}^{+}$such that $\liminf _{\varepsilon \rightarrow 0} \psi(\varepsilon)>0$ holds. We will see at the end of the section that a reasonable choice is $\psi(\varepsilon)=\varepsilon^{-n \theta}$ for some $\theta>0$. Let us write $\mathcal{M}_{\ell}$ as a sum of two 
operators $\mathcal{M}_{\ell, \varepsilon}$ and $V_{\ell, \varepsilon}(\operatorname{see}(42))$ :

$$
\begin{aligned}
\left\langle\mathcal{M}_{\ell, \varepsilon}\left(u_{1}, \ldots, u_{n}\right) u_{n+1}, u_{0}\right\rangle & =\sum_{\substack{\Omega_{n+2}(\ell)^{c} \\
k_{0}^{\alpha^{\prime}} \leq \psi(\varepsilon)}} m_{\ell}\left(k_{0}, \ldots, k_{n+1}\right) \prod_{j=0}^{n+1}\left\langle u_{j}, \phi_{\alpha, k_{j}}\right\rangle \\
\left\langle V_{\ell, \varepsilon}\left(u_{1}, \ldots, u_{n}\right) u_{n+1}, u_{0}\right\rangle & =\sum_{\substack{\Omega_{n+2}(\ell)^{c} \\
k_{0}^{\alpha^{\prime}}>\psi(\varepsilon)}} m_{\ell}\left(k_{0}, \ldots, k_{n+1}\right) \prod_{j=0}^{n+1}\left\langle u_{j}, \phi_{\alpha, k_{j}}\right\rangle .
\end{aligned}
$$

Remember that the $\mathcal{H}_{\alpha}^{s}$ boundedness holds for $\mathcal{M}_{\alpha, n, \delta}^{2 s-\frac{1}{\alpha}, \nu}$, then writing

$$
k_{0}^{\alpha^{\prime}(2 s-2)} \leq \frac{k_{0}^{\alpha^{\prime}\left(2 s-\frac{1}{\alpha}\right)}}{\psi(\varepsilon)^{2-\frac{1}{\alpha}}}
$$

allows us to to claim that

$$
\left|\left\langle V_{\ell, \varepsilon}\left(u_{1}, \ldots, u_{n}\right) u_{n+1}, u_{0}\right\rangle\right| \leq \frac{C}{\psi(\varepsilon)^{2-\frac{1}{\alpha}}} \prod_{j=0}^{n+1}\left\|u_{j}\right\|_{\mathcal{H}_{\alpha}^{s}} .
$$

The term $\mathcal{M}_{\ell, \varepsilon}$ will be eliminated by a normal form procedure.

Proposition 5.4. - We suppose that $\alpha$ equals 2 or 3 . For any $s$ enough large, $\rho>0$ and $\varepsilon>0$, there are $\nu_{0}>0$ and

$$
\underline{\mathcal{M}_{\ell, \varepsilon}} \in \mathcal{M}_{\alpha, n, \delta}^{2 s-\frac{1}{\alpha}, \nu+\nu_{0}}[\ell]
$$

such that for any $\mathcal{H}_{\alpha}^{s}$ strong solution $u$ of $\dot{u}=i \Lambda_{\alpha, m} u-i w^{n+1}$, one has

$$
\begin{aligned}
& \frac{d}{d t}\left\langle\underline{\mathcal{M}_{\ell, \varepsilon}}(\bar{u}, \ldots, \bar{u}, u, \ldots, u) u, u\right\rangle \\
= & \left\langle\mathcal{M}_{\ell, \varepsilon}(\bar{u}, \ldots, \bar{u}, u, \ldots, u) u, u\right\rangle+\psi(\varepsilon)^{\frac{3}{\alpha}-1+\rho} \mathcal{O}\left(\|u\|_{\mathcal{H}_{\alpha}^{s}}^{2 n+2}\right) .
\end{aligned}
$$

Proof. - We make the same reasoning that the one in the proof of Proposition 5.2. We define

$$
\left.\underline{\left\langle\mathcal{M}_{\ell, \varepsilon}\right.}\left(u_{1}, \ldots, u_{n}\right) u_{n+1}, u_{0}\right\rangle=\sum_{\substack{\Omega_{n+2}(\ell)^{c} \\ k_{0}^{\alpha^{\prime}}<\psi(\varepsilon)}} \frac{m_{\ell}\left(k_{0}, \ldots, k_{n+1}\right)}{-i F_{m}^{\ell}\left(\lambda_{\alpha, k_{0}}, \ldots, \lambda_{\alpha, k_{n+1}}\right)} \prod_{j=0}^{n+1}\left\langle u_{j}, \phi_{\alpha, k_{j}}\right\rangle .
$$

Using (18) and the trivial inequality

$$
k_{0}^{\alpha^{\prime}\left(2 s-2+1+\frac{2}{\alpha}+\rho\right)} \leq k_{0}^{\alpha^{\prime}\left(2 s-\frac{1}{\alpha}\right)} \psi(\varepsilon)^{\frac{3}{\alpha}-1+\rho} .
$$

The $\mathcal{H}_{\alpha}^{s}$ boundedness property proves that

$$
\left|\left\langle\mathcal{M}_{\ell, \varepsilon}\left(u_{1}, \ldots, u_{n}\right) u_{n+1}, u_{0}\right\rangle\right| \leq C \psi(\varepsilon)^{\frac{3}{\alpha}-1+\rho} \prod_{j=0}^{n+1}\left\|u_{j}\right\|_{\mathcal{H}_{\alpha}^{s}} .
$$


When we compute the derivative of $\left\langle\mathcal{M}_{\ell, \varepsilon}(\bar{u}, \ldots, \bar{u}, u, \ldots, u) u, u\right\rangle$, we first get

$$
\left\langle\mathcal{M}_{\ell, \varepsilon}(\bar{u}, \ldots, \bar{u}, u, \ldots, u) u, u\right\rangle
$$

as in the proof of Proposition 5.2, and then we get $n+2$ terms which involve the nonlinearity $w^{n+1}$, for instance

$$
\left.\underline{\left\langle\mathcal{M}_{\ell, \varepsilon}\right.}(\bar{u}, \ldots, \bar{u}, u, \ldots, u) u, w^{n+1}\right\rangle .
$$

We can conclude because $\left\|w^{n+1}\right\|_{\mathcal{H}_{\alpha}^{s}} \leq C\|u\|_{\mathcal{H}_{\alpha}^{s}}^{n+1}$.

The proof of Theorem 1.3 is then quite similar. For $s$ enough large, if $u$ is a $\mathcal{H}_{\alpha}^{s}$ strong solution of $\dot{u}=i \Lambda_{\alpha, m} u-i w^{n+1}$ then define

$$
\begin{aligned}
& M_{s, \varepsilon}(u, \ldots, u) \\
:= & \operatorname{Re} i \sum_{\ell=0}^{n}\left[\left\langle\underline{\mathcal{M}_{\ell, \varepsilon}}(\underbrace{\bar{u}, \ldots, \bar{u}}_{\ell}, \underbrace{u, \ldots, u}_{n-\ell}) u, u\right\rangle+\langle\underline{\widehat{\mathcal{M}}_{\ell}} \underbrace{\bar{u}, \ldots, \bar{u}}_{\ell}, \underbrace{u, \ldots, u}_{n-\ell}) \bar{u}, u\right\rangle \\
& \left.+\left\langle\underline{\mathcal{R}_{\ell}}(\underbrace{\bar{u}, \ldots, \bar{u}}_{\ell}, \underbrace{u, \ldots, u}_{n-\ell}) u\right\rangle+\left\langle\underline{\widehat{\mathcal{R}}_{\ell}}(\underbrace{\bar{u}, \ldots, \bar{u}}_{\ell}, \underbrace{u, \ldots, u}_{n-\ell}) \bar{u}, u\right\rangle\right] .
\end{aligned}
$$

The inequality (45), Proposition 4.1, Proposition 5.4 and and the part of Proposition 5.2 which involves $\underline{\widehat{\mathcal{M}}_{\ell}}, \underline{\mathcal{R}_{\ell}}$ and $\underline{\widehat{\mathcal{R}}_{\ell}}$ imply the following fact

$$
\begin{aligned}
& \frac{d}{d t}\left[\Theta_{s}(u)-M_{s}(u, \ldots, u)\right] \\
= & \left.\psi(\varepsilon)^{\frac{3}{\alpha}-1+\rho} \mathcal{O}\left(\|u\|_{\mathcal{H}_{\alpha}^{s}}^{2 n+2}\right)+\sum_{\ell=0}^{n} \operatorname{Re} i V_{\ell, \varepsilon}(\underbrace{\bar{u}, \ldots, \bar{u}}_{\ell}, \underbrace{u, \ldots, u}_{n-\ell}) u, u\right\rangle \\
= & \psi(\varepsilon)^{\frac{3}{\alpha}-1+\rho} \mathcal{O}\left(\|u\|_{\mathcal{H}_{\alpha}^{s}}^{2 n+2}\right)+\frac{1}{\psi(\varepsilon)^{2-\frac{1}{\alpha}}} \mathcal{O}\left(\|u\|_{\mathcal{H}_{\alpha}^{s}}^{n+2}\right) .
\end{aligned}
$$

We have used that $\liminf _{\varepsilon \rightarrow 0} \psi(\varepsilon)>0$ holds to include the $\mathcal{O}\left(\|u\|_{\mathcal{H}_{\alpha}^{s}}^{2 n+2}\right)$ contributions of $\widehat{\mathcal{M}}_{\ell}, \underline{\mathcal{R}_{\ell}}$ and $\widehat{\mathcal{R}}_{\ell}$ in the left part of (46). Notice that $\frac{3}{\alpha}-1+\rho$ is positive since $\alpha \in\{2,3\}$. Now remember that if $\|u\|_{\mathcal{H}_{\alpha}^{s}}=\varepsilon$ is enough small then obviously $\Theta_{s}(u) \simeq \Theta_{s}(u)-M_{s, \varepsilon}(u, \ldots, u) \simeq \varepsilon^{2}$ holds (because $\underline{\mathcal{M}}_{\ell, \varepsilon}$ has the $\mathcal{H}_{\alpha}^{s}$ boundedness property, see Proposition 5.4). Finally, if the initial data $u(0, \cdot)$ of $\dot{u}=i \Lambda_{\alpha, m} u-i w^{n+1}$ is of order $\varepsilon$ in $\mathcal{H}_{\alpha}^{s}$ and if $T>0$ is the largest time such that $\sup \|u(t, \cdot)\|_{\mathcal{H}_{\alpha}^{s}} \leq K \varepsilon$ then (46) allows us to write

$$
K^{\prime} \varepsilon^{2} \leq T \varepsilon^{2+n}\left(\varepsilon^{n} \psi(\varepsilon)^{\frac{3}{\alpha}-1+\rho}+\frac{1}{\psi(\varepsilon)^{2-\frac{1}{\alpha}}}\right)
$$


We just have to choose an adequate map $\psi$ to get a maximal $T$. Notice whatever is the choice of $\psi$, we always have

$$
\begin{aligned}
&\left(\varepsilon^{n} \psi(\varepsilon)^{\frac{3}{\alpha}-1+\rho}\right)^{\frac{2-\frac{1}{\alpha}}{1+\frac{2}{\alpha}+\rho}\left(\frac{1}{\psi(\varepsilon)^{2-\frac{1}{\alpha}}}\right)^{\frac{\frac{3}{\alpha}-1+\rho}{1+\frac{2}{\alpha}-\rho}}} \leq C_{\alpha, \rho}\left(\varepsilon^{n} \psi(\varepsilon)^{\frac{3}{\alpha}-1+\rho}+\frac{1}{\psi(\varepsilon)^{2-\frac{1}{\alpha}}}\right) \\
& \varepsilon^{n\left(\frac{2-\frac{1}{\alpha}}{1+\frac{2}{\alpha}+\rho}\right)} \leq C_{\alpha, \rho}\left(\varepsilon^{n} \psi(\varepsilon)^{\frac{3}{\alpha}-1+\rho}+\frac{1}{\psi(\varepsilon)^{2-\frac{1}{\alpha}}}\right) .
\end{aligned}
$$

Therefore, a reasonable choice for $\psi(\varepsilon)$ is $\varepsilon^{\frac{-n}{1+\frac{2}{\alpha}+\rho}}$. And we get

$$
\varepsilon^{-n\left(\frac{3 \alpha+1+\alpha \rho}{\alpha+2+\alpha \rho}\right)}=\varepsilon^{-n\left(\frac{2-\frac{1}{\alpha}}{1+\frac{2}{\alpha}+\rho}\right)-n} \leq T .
$$

Since $\rho>0$ can be chosen arbitrary small, plugging $\alpha=2$ or $\alpha=3$ gives the conclusion. Theorem 1.3 is proved.

The author would like to thank Qidi Zhang and Jean-Marc Delort for valuable discussions.

\section{BIBLIOGRAPHY}

[Bam03] D. BAmBusi - "Birkhoff normal form for some nonlinear PDEs", Comm. Math. Physics 234 (2003), p. 253-285.

[Bam07] D. BAmbusi - "A Birkhoff normal form theorem for some semilinear pdes", in Hamiltonian Dynamical Systems and Applications, Springer, 2007, p. 213-247.

[BDGS07] Bambusi, Delort, Grébert \& Szeftel - "Almost global existence for Hamiltonian semilinear Klein-Gordon equations with small Cauchy date on Zoll manifolds", Comm. Pure Appl. Math 60 no 11 (2007), p. pages 1665-1690.

[BG04] D. Bambusi \& B. GRÉBERT - "Forme normale pour NLS en dimension quelconque", Compt. Rendu. Acad. Sciences Paris (2004).

[BG06] D. BAmbusi \& B. GRÉBERT - "Birkhoff normal form for PDEs with tame modulus", Duke Math. J. 135 (2006), p. 507-567.

[Bou96] J. BourgaIN - "Construction of approximative and almost periodic solutions of perturbed linear schrödinger and wave equations", Geometric and Functional Analysis 6 (1996), no. 2, p. 201-230.

[BS91] F. BEREZIN \& M. SHUBIN - The Schrödinger equation, vol. 66, Springer, 1991.

[Del09] J. DelorT - "On long time existence for small solutions of semilinear Klein-Gordon equations on the torus", Journal d'Analyse Mathématique 107 (2009), no. 1, p. 161-194. 
[DS04] J. M. DELORT \& J. SzEFTEL - "Long-time existence for small data nonlinear Klein-Gordon equations on tori and spheres", Internat. Math. Res. Notices 37 (2004), p. 1897-1966.

[DS06a] _ _Long-time existence for semi-linear Klein-Gordon equations with small cauchy data on Zoll manifolds", Amer. J. Math 128 (2006), p. 1187-1218.

[DS06b] J. Delort \& J. Szeftel - "Bounded almost global solutions for non hamiltonian semi-linear Klein-Gordon equations with radial data on compact revolution hypersurfaces", Annales de l'Institut Fourier 56 (2006), no. 5, p. 1419-1456.

[FZ10] D. FANG \& Q. ZHANG - "Long-time existence for semi-linear kleingordon equations on tori", Journal of Differential Equations 249 (2010), no. 1, p. 151-179.

[GIP09] B. GrÉbert, R. Imekraz \& E. PAturel - "Normal forms for semilinear quantum harmonic oscillators", Comm. Math. Phys. vol 291-3 (2009), p. pages 763-798.

[Gré07] B. GRÉBERT - "Birkhoff normal form and Hamiltonian PDEs", in Partial differential equations and applications, Sémin. Congr., vol. 15, Soc. Math. France, Paris, 2007, p. 1-46.

[HR82] B. HELFFER \& D. RoBerT - "Asymptotique des niveaux dénergie pour des hamiltoniens à un degré de liberté", Duke mathematical journal 49 (1982), no. 4, p. 853-868.

[Ime12a] R. IMEKRAZ - "Condition de non-résonance pour l'oscillateur harmonique quantique perturbé", Dynamics of PDE, vol 9, number 3 (2012), p. 205-238.

[Ime12b] _ "Nomal forms for semilinear superquadratic oscillators", Journal of differential equations vol 252 (2012), p. pages 20252052 .

[Ime13] _ _ "Existence en temps grand pour des équations de KleinGordon à petite donnée initiale sur une structure de Toeplitz", J. Funct. Anal. 264 (2013), no. 6, p. 1480-1524.

[RS78] M. ReEd \& B. Simon - "Analysis of operators, vol. IV of methods of modern mathematical physics", 1978.

[YZ04] K. YAJIMA \& G. ZHANG - "Local smoothing property and Strichartz inequality for Schrdinger operator with potentials superquadratic at infinity", Journal of differential equations 202 (2004), p. 81-110.

[Zha10] Q. ZHANG - "Long-Time Existence for Semi-Linear Klein-Gordon Equations with Quadratic Potential", Communications in Partial Differential Equations 35 (2010), no. 4, p. 630-668. 